\title{
Theorieatmosphären. Soziologische Denkstile als affektive Praxis
}

\author{
Elena Beregow
}

Angenommen: 11. August 2021 / Online publiziert: 6. September 2021

(C) Der/die Autor(en) 2021

Zusammenfassung Dieser Artikel führt das Konzept der Theorieatmosphäre ein, um die affektive Dimension von Theorien zu adressieren. Jenseits einer Engführung auf die Autor:in oder auf den Text wird die Theorieatmosphäre als Produkt des performativen Zusammenwirkens von Text, Lesepraktiken, Dingen und Räumen entworfen. Im Anschluss an Ludwik Flecks Theorie der Denkstile, Denkkollektive und Stimmungen wird die Funktionsweise von Theorieatmosphären umrissen und mithilfe der jüngeren Debatten um Atmosphären und Affekte aktualisiert. In dieser Weise können Theorieatmosphären als Produkte affektiver Praxis (Wetherell) verstanden und um die Rolle nichtmenschlicher Elemente erweitert werden. In einem Vergleich des systemtheoretischen (Luhmann) und des dekonstruktiven Denkstils (Deleuze und Guattari) erfolgt anhand der Parameter des Textes, der Praxis des Lesens sowie der Dinge und Räume von Theorie eine Analyse zweier konträrer Theorieatmosphären. Ausgehend von den Selbstbeschreibungen und den dabei wichtigen metaphorischen Registern werden diese als „heiße“ vs. „kalte“ Theorien akzentuiert. Das Konzept der Theorieatmosphäre zielt auf eine konstitutive, aber meist übersehene Dimension von Theorien und möchte zur Debatte zu Prozessen des Theoretisierens sowie von Schulbildungs- und Rezeptionsprozessen beitragen, indem es erste Antworten auf die Frage liefert: Warum faszinieren Theorien?

Schlüsselwörter Denkstil · Denkkollektiv · Atmosphäre · Affekt · Stimmung · Theoretisieren $\cdot$ Affektive Praxis

Elena Beregow $(\bowtie)$

Professur für Allgemeine Soziologie und Soziologische Theorie, Universität der Bundeswehr München, Werner-Heisenberg-Weg 39, 85579 Neubiberg, Deutschland

E-Mail: elena.beregow@unibw.de 


\title{
Atmospheres of theory. Sociological thought styles as affective practices
}

\begin{abstract}
This article introduces the concept of "atmospheres of theory" in order to address the affective dimension of theories. Beyond a narrow focus on the author or the text, the atmosphere of theory is conceived of as a product of the performative interactions between text, reading practices, things and spaces. Following Ludwik Fleck's theory of thought styles, thought collectives and moods, the dynamics of theoretical atmospheres are outlined and updated by means of the recent debates on atmospheres and affects. In this way, theoretical atmospheres can be grasped as products of affective practice (Wetherell) and amended with regards to non-human objects. By comparing the systems-theoretical (Luhmann) and the deconstructive thought style (Deleuze and Guattari), the article presents an analysis of two contradicting theoretical atmospheres considering the parameters of the text, the practice of reading, and the things and spaces of theory. Based on the self-descriptions and important metaphorical registers, they are accentuated as "hot" vs. "cold" theories. The concept of theoretical atmospheres targets a constitutive but mostly overlooked dimension of theories and serves to contribute to the debate about processes of theorizing by offering tentative answers to the question: Why do theories fascinate?
\end{abstract}

Keywords Thought collective $\cdot$ Thought style Atmosphere $\cdot$ Mood Affect · Theorizing $\cdot$ Affective practice

\section{Atmosphère théoriques. Les styles de pensée sociologiques comme praxis affective}

Résumé Cet article introduit le concept d'atmosphère théorique pour appréhender la dimension affective des théories. Au-delà d'une réduction à l'auteur.e ou au texte, l'atmosphère théorique est conçue comme le produit de l'interaction performative entre texte, pratiques de lecture, objets et espaces. Dans le prolongement de la théorie des styles de pensée, des collectifs de pensée et des ambiances de Ludwik Fleck, le fonctionnement des atmosphères théoriques est esquissé et actualisé en tirant parti des débats récents sur les atmosphères et les affects. Les atmosphères théoriques peuvent être ainsi conçues comme les produits d'une praxis affective (Wetherell) et élargies de manière à intégrer le rôle des éléments non humains. À travers une comparaison entre les styles de pensée de la théorie des systèmes (Luhmann) et de la déconstruction (Deleuze et Guattari), une analyse de deux atmosphères théoriques opposées est opérée suivant les paramètres du texte, de la pratique de la lecture ainsi que des objets et des espaces de la théorie. À partir des descriptions de soi et des registres métaphoriques qui jouent un rôle important dans ce contexte, ces théories sont qualifiées de «chaude» et de «froide». Le concept d'atmosphère théorique vise une dimension constitutive mais généralement ignorée des théories et contribue au débat sur les processus de théorisation ainsi que sur les processus d'éducation scolaire et de réception en livrant de premières réponses à la question: Pourquoi les théories fascinent-elles? 
Mots-clés Style de pensée · Collectif de pensée $\cdot$ Atmosphère $\cdot$ Affect · Ambiance · Théorisation · Praxis affective

\section{Einleitung}

Texte haben eine Atmosphäre. Diese Annahme wird einem bei literarischen Texten, bei Romanen und Gedichten schnell einleuchten: Wir mögen die düster-beklemmende Atmosphäre der Krimis von Edgar Wallace wahrnehmen, die theatral aufgeladene Atmosphäre von Popliteratur auf uns wirken lassen oder die surreal-sedierte Atmosphäre des Werks von Marcel Proust goutieren. Dieser Beitrag geht der Intuition nach, dass auch soziologische Theorien an Atmosphären gebunden sind, die sich entscheidend auf ihre Zirkulation und Rezeption auswirken - Atmosphären, die nicht nur im Text zu suchen sind, sondern sich erst im Verbund mit bestimmten Lesepraktiken, Dingen und Räumen formieren.

Die meisten soziologischen und kulturwissenschaftlichen Atmosphärenanalysen richten ihren Fokus auf Phänomene wie Kunstwerke (Wagner 2007; Rauh 2012), Grabfeiern (Wetherell 2013) oder rituellen Kirchengesang (Riedel 2015). Nimmt man aber die vielbeschworene Wendung der „Affektivität des Sozialen“ (Reckwitz 2016, S. 167) ernst, dann müsste es darum gehen, nicht nur gezielt auf affektivästhetisches Erleben hin ausgerichtete Phänomene auf ihre Affektdynamiken hin zu untersuchen, sondern auch solche, die in Selbst- wie Fremdbeschreibungen auBerhalb des Affektiven verortet werden. Denn während etwa literarische Texte ihre narrativen und stilistischen Möglichkeiten experimentell ausreizen, um bewusst Atmosphären zu konstruieren, läuft eine solche Ambition dem klassischen Theorieideal zuwider. Das ernsthafte Verständnis ,reiner Theorie“, das ihr lange den Ruf der Königsdisziplin des Fachs einbrachte, scheint noch von der Idee eines „Abstractus“ umweht, das im Hochmittelalter keine Eigenschaft des Textes bezeichnete, sondern einen Gemütszustand seines Autors. Frei von allen sinnlichen Wahrnehmungen, affektiven und sozialen Bindungen stand dieser für die ideale Konzentration eines Gelehrten, sein „Klarsehen in Trance“ (Groebner 2012, S. 115). Weil Abstraktion bis heute geradezu als Alleinstellungsmerkmal von Theorie gilt, überrascht es kaum, dass Theoretiker:innen dazu neigen, die Produktion von Atmosphären - die im Wort „Trance“ deutlich durchklingt - zu bestreiten oder allenfalls als unbedeutendes Dekorum abtun. Auch soziologische Theorietexte sind aus dieser Sicht ästhetische Texte wider Willen.

Um die Atmosphäre von Texten systematisch zu beleuchten, stehen zunächst zwei bewährte Wege zur Verfügung, deren jeweilige Probleme jedoch, wie zu zeigen, einen dritten notwendig machen. Erstens ist dies der Ansatz der Rhetorikanalyse, der Inspirationen aus literaturwissenschaftlichen Verfahren schöpft, zweitens die Soziologie des Charismas im Anschluss an Max Weber. In rhetorikanalytischer Tradition hat Hans Ulrich Gumbrecht (2011) vor einigen Jahren die Stimmung als neues Paradigma der Literaturwissenschaften ausgerufen. Die Stimmungsqualitäten literarischer Texte, so sein ästhetisches Argument, enthalten ein objektivierbares atmosphärisches Echo ihres jeweiligen Zeitgeistes, das mithilfe einer physisch-affektiv nachspürenden Lektüreweise wieder eingefangen werden kann. Begreift man 
die Atmosphäre als Bestandteil der Form eines Textes, so lässt sich Gumbrechts Idee des „Stimmungen lesen“ als eine Methode einordnen, die über die Mikroatmosphäre eines Textes zur Makroatmosphäre seines historisch-sozialen Kontexts vordringt. Ausgehend von der atmosphärischen Form eines Textes - seiner „Oberfläche“ gelangt sie zu seiner inhaltlichen Bedeutung - seiner „Tiefe“.

Soziologische Anschlüsse an Rhetorikanalysen, die sich dem Interesse an der formalen Ebene soziologischer Theorien verdanken, haben gezeigt, dass Theorien aufgrund ihres Abstraktionsgrads auf Metaphern, Exempla und Gründungsszenen angewiesen sind, um Evidenz zu erzeugen (Lüdemann 2004; Farzin 2011; Farzin und Laux 2014; Schlechtriemen 2014). Würde man diesen soziologischen Pfad atmosphärologisch weiterverfolgen, dann erschienen die Atmosphären als Ergebnis rhetorischer Figuren, die es auf der immanenten Ebene des Textes herauszuarbeiten gälte. Die Stärke solcher formalen Analysen markiert im Fall der Atmosphäre aber zugleich ihre Grenze: Eine Analyse, die sich auf den Text beschränkt, muss die übertextlichen Aspekte seiner atmosphärischen Wirkung übersehen.

Der zweite Weg geht über den Text hinaus, verengt die atmosphärische Wirkung von Theorie aber auf die Präsenz der Theoretiker:in. Demnach lässt sich die Wirkung von Theorieatmosphären an der Faszination ablesen, die die Protagonist:innen einer Theorie auf Außenstehende ausüben. Diese Faszination liegt auch dem Charismabegriff zugrunde, den Max Weber (1980, S. 140 ff.) u.a. auch in Auseinandersetzung mit künstlerischen Sekten wie dem George-Kreis entwickelte, die über die Präsenz eines kultisch verehrten „Meisters“ zusammengehalten werden. Ausgehend von Weber bestimmt Marko Juvan das ,charisma of theory“ als die faszinierende personale Präsenz des allwissenden Theoretikers als ,subject supposed to know“ (Juvan 2018, S. 89). Weniger wissens- als vielmehr kultursoziologisch verfolgt auch Bartmanski (2012) die Frage „How to become an iconic social thinker“ und arbeitet die symbolisch-performativen Prozesse heraus, die Sozialtheoretiker zu Ikonen, Klassikern oder „Gründungsvätern“ machen.

Angesichts eines langsamen, aber sicheren Rückgangs des klassischen Meisterdiskurses hat das Atmosphärenkonzept den schlagenden Vorteil, einerseits die affektiven Wirkungen zu erfassen, die von einzelnen Theoriefiguren ausgehen, diese aber andererseits auch in ihren nichtpersonalen Dimensionen zu verstehen. Umgekehrt erschöpfen sich diese Wirkungen, anders als soziologische Rhetorikanalysen nahelegen, nicht in der form- bzw. textzentrierten Demaskierung der Evokationsprinzipien von Stil und Aura. Vielmehr adressiert das Konzept der „Theorieatmosphäre“ den verbindenden Raum des „Dazwischen“, der nicht nur Autor:in und Text, sondern auch Lese- und Rezeptionspraktiken sowie Dinge und Räume des konkreten Gebrauchs von Theorie umspannt, wechselseitig verklammert und affektiv zueinander ins Verhältnis setzt. So sehr etwa die atmosphärische Wirkung der LuhmannSpezies $^{1}$ gewiss von der Theoretikerfigur Luhmann und den Luhmann'schen Texten geprägt ist, so deutlich zeigen die Systemtheoretiker:innen sich bei näherem Hinse-

\footnotetext{
1 Ein Cartoonist bilanzierte nach einer Luhmann-Tagung in Bielefeld: „Diese Tagung, diesen Zoo voller komischer Vögel, meist mit männlicher Befiederung zu beobachten und deren Verhalten zu studieren, war die eigentliche ,Einführung in die Systemtheorie der Gesellschaft ‘ [...]. Diese Luhmann-Spezies vorgeführt zu bekommen war weit aufschlussreicher als alles, was diese fachlich von sich zu geben hatte." (Lierschof 2017) Ob man nun pastoral anmutende Luhmannianer in Tweedjacketts vor sich hat, melan-
} 
hen als Produzent:innen einer spezifischen Theorieatmosphäre, die sich gegenüber der Bezugsfigur des Meisterdenkers verselbstständigt hat.

Ausgehend von dieser Beobachtung möchte ich den „dritten Weg“ jenseits von Autoren- bzw. Werkzentrierung im Anschluss an Ludwik Flecks wissenschaftssoziologische Arbeiten skizzieren. Mit dem „Denkkollektiv“ entwarf Fleck in den 1930erJahren ein Konzept, wonach wissenschaftliche Theorien auf einem je spezifischen „Denkstil“ und einer damit einhergehenden „Stimmung“ beruhen. Damit liegt ein geeigneter Einsatzpunkt vor, um eine analytische Perspektive auf Theorieatmosphären innerhalb der Soziologie zu entwickeln: Mit Fleck kommen die interaktiven Herstellungsweisen einer bestimmten Theorieatmosphäre jenseits ihrer einfachen Rückführung auf eine Autor:in oder einen Textkorpus in den Blick. Im Lichte der aktuellen affekttheoretischen Debatte wirkt Flecks subjektzentrierter Fokus auf das „Gefühl“ jedoch korrekturbedürftig. Daher wird im Folgenden von Affekten statt von Gefühlen, von Atmosphären statt von Stimmungen ausgegangen.

Bei Theorieatmosphären handelt es sich, so die leitende These der folgenden Überlegungen, nicht nur um illustratives Beiwerk, sondern um eine konstitutive Dimension von Theorien - und zwar keineswegs nur solcher, die „stimmungsvoll““ daherkommen. Mehr noch: Es gibt keine Theorie ohne Atmosphäre. Selbst der Versuch, möglichst schnörkellos zu schreiben und auf unnötige Stilblüten zu verzichten, wird je nach Kontext erst recht eine möglicherweise besonders provokante Atmosphäre hervorbringen. Als materialisierte Zwischen- und Randbereiche der Theorie laufen Atmosphären meist beiläufig und unsichtbar mit, drängen sich aber bisweilen exzessiv in den Vordergrund. Theorieatmosphären möchte ich im Folgenden verstanden wissen als Produkte textlicher und außertextlicher Elemente, die den Schreibund Leseprozess sowie die Wahrnehmung von Theorie auf affektive Weise initiieren, ordnen, gestalten, steuern, rahmen und ausrichten.

Der Mehrgewinn dieser zunächst recht breit angelegten Perspektive besteht darin, textliche und außertextliche Formen in ihrem Zusammenspiel sichtbar machen zu können. Wenn von Theorieatmosphären die Rede ist, dann lässt sich das lesende, schreibende und verstehende Subjekt zwar nicht einfach ausblenden. Und doch sind hier gerade die Momente von Interesse, in denen wir diese Tätigkeiten nicht als Ausprägungen eines reflexiven Bewusstseins, sondern als Resultat kollektiver Denkstile begreifen können. „Theorieatmosphäre“ meint daher nicht nur eine hochgradig wirksame und häufig übersehene Formdimension theoretischer Texte, sondern ein verschränktes Produkt von Lesepraktiken, der damit verbundenen Zirkulation von Dingen und der Konstruktion von Räumen, deren Materialität umgekehrt auf die textuelle Funktionsweise von Theorien zurückwirkt. Von Interesse ist das ,Dazwischen“, in dem diese heterogenen Dinge und Akteure durch kollektive Praktiken verbunden werden. Die Analyse fragt also danach, wie Theorie als gleichermaßen ästhetischer, d.h. sinnlicher und affektiver, wie denkstilspezifischer und damit so-

cholische Adorniten, die am nachgeschobenen ,sich“ festhalten, oder wild assoziierende Deleuzianer: Von diesen selbst als außertheoretisch wahrgenommenen Umgebungen der Theorie scheint ein großer Reiz auszugehen. Verirren sich Journalist:innen oder gutwillige Studierende auf Tagungen, staunen sie nicht selten über die Schrullen des verschworenen Theoriekollektivs, dem seine seltsamen Stilblüten und habituellen Eigentümlichkeiten in der Regel ebenso entgehen wie der spezifische Sound, der innerhalb dieses Kollektivs entsteht. 
zialer Vollzug - kurzum: als affektive Praxis - funktioniert. Entgegen der Tendenz, Theorie als immateriell-vergeistigten Vorgang zu mystifizieren, soll auf diese Weise ihre affektive Strahlkraft verstehbar werden.

Innerhalb der Soziologie hat in jüngerer Zeit eine Debatte um das Theoretisieren Auftrieb erhalten, die auf das Desiderat einer empirischen Erforschung von Theoriearbeit reagiert (Abbott 2004; Swedberg 2014). Das Konzept der Theorieatmosphäre möchte zu dieser Empirisierung der soziologischen Theoriearbeit beitragen. Theorien sind dabei weniger als ,,beobachtungsleitende Annahmen“ zu verstehen, sondern vielmehr als „,beobachtbare soziale Phänomene, die die Welt mit erzeugen, die sie beschreiben." (Kalthoff 2008, S. 13) Es kann also nicht darum gehen, Theorien an übergeordnete Kriterien wie Falsifizierbarkeit zu knüpfen, sondern es ist - ganz im Sinne Flecks - von Definitions- und Deutungskämpfen innerhalb konkurrierender Theoriefelder, von Prozessen der fachinternen Übersetzung, der (Wieder-)Entdeckung, der Kanonisierung und des Vergessens abhängig, welche Arbeiten unter dem Banner ,soziologische Theorie“ rangieren dürfen und welche nicht. Was (soziologische) Theorie ist, wird damit selbst zur empirischen Frage. Beispielsweise ist es noch nicht lange her, dass Deleuze - nicht zuletzt im Rahmen der Affektdiskussion als Autor der soziologischen Theoriebildung lesbar gemacht wurde (vgl. dazu auch Delitz 2015; Seyfert 2019).

Im Folgenden werden erstens die Begriffe „Denkstil“, „Denkkollektiv“ und „Stimmung“ im Sinne Flecks umrissen (Abschnitt 2). Zweitens wird der Begriff der „Atmosphäre“ skizziert, theoretisch verortet und zum Affektbegriff ins Verhältnis gesetzt. Um Flecks analytische Erträge weiterzuführen, werde ich diese mithilfe von Margaret Wetherells Begriff der ,affektiven Praktiken“ präzisieren (Abschnitt 3). Drittens folgt eine kurze Arbeitsdefinition von „Theorieatmosphäre“, exemplarisch vergleichend untersucht anhand des „luhmannianischen“ und „deleuzianischen“ Denkstils mit besonderem Fokus auf die bis dato vor allem von Felsch (2015) untersuchte westdeutsche Theorierezeption seit den 1970er-Jahren (Abschnitt 4).

\section{Denkkollektiv, Denkstil, Stimmung: Grundlegung eines soziologischen Atmosphärenbegriffs mit Fleck}

1935 legte der polnische Mediziner, Philosoph und Wissenssoziologe Ludwik Fleck eine Studie zur Entstehung und Entwicklung einer wissenschaftlichen Tatsache (Fleck 1980 [1935]) vor, in der er die Begriffe des „Denkstils“ und des „Denkkollektivs“" einführte. ${ }^{2}$ Wissen, und damit Theorie, so Flecks Grundgedanke, ist immer auch das Resultat einer sozialen ,,Tätigkeit, veranstaltet von Forschergemeinschaf-

\footnotetext{
2 Über fast drei Jahrzehnte wurden Flecks wissenschaftssoziologische Thesen auch in der Soziologie kaum rezipiert. Das änderte sich indes schlagartig mit der Erwähnung Flecks in einer Fußnote im Vorwort von Thomas S. Kuhns Werk The Structure of Scientific Revolutions (1962). Mittlerweile, so die Einschätzung Egloffs, stehe Fleck, ,im Begriff, seinen einstigen (Wieder-)Entdecker als Klassiker (post-)moderner Wissenschaftsgeschichte und -soziologie in den Schatten zu stellen“ (Egloff 2005, S. 7). Denn während Wissenschaft bei Kuhn von anderen gesellschaftlichen Bereichen isoliert wird, denkt Fleck Wissensproduktion als genuin sozialen Prozess - und gibt damit nicht zuletzt auch Aufschluss über ihre kollektive Produktion sowie die „Historizität wissenschaftlichen Wissens“ (Rheinberger 2005).
} 
ten“ (Schäfer und Schnelle 1980, S. VII). Ein Denkkollektiv ist nicht einfach die Summe zweier Denkender und ihrer individuellen Gedanken, sondern beruht auf der spezifischen „Stimmung“, die als Zwischenraum das Denkvermögen der Einzelnen vermittelt, schöpferisch steigert und verändert. Die Stimmung definiert Fleck als „Bereitschaft für selektives Empfinden und für entsprechend gerichtetes Handeln“ (Fleck 1980 [1935], S. 130). Das Resultat dieser Bereitschaft ist ein „Denkgebilde“, dem weder mit individualistischen Erkenntnistheorien noch mit dem klassischen Ideal der Autorschaft beizukommen ist, weil es nicht auf die gedankliche Leistung Einzelner oder Mehrerer, sondern auf ,die besondere Stimmung“ zwischen ihnen zurückgeht. Auch wenn die Singularität der Stimmung für Fleck nicht das Werk der Teilnehmenden des Denkkollektivs ist - und auch nicht ihrer schlichten Addition -, so bedarf es doch deren konkreter Präsenz: Mit jedem neuen Teilnehmenden entsteht ein neues Kollektiv und mit diesem eine neue, ihrerseits ,besondere“ Stimmung. ${ }^{3}$

Die Stimmung versteht Fleck als eine energetische Kategorie der situativen Präsenz, weshalb er sie auch mit dem „Kraftfeldbegriffe der Physik“ vergleicht. Anders als ontologisch gefärbte Atmosphärenbegriffe nahelegen, handelt es sich um einen „mehr funktionellen als substanziellen Begriff“ (ebd., S. 135), insofern es um die konkreten Wirkungen der Erzeugung von Denktätigkeit geht. Kommt es zu einer Übereinstimmung zwischen zwei Personen, dann ist laut Fleck ,,[d]iese Stimmungskameradschaft [...] sofort, nach einigen Sätzen fühlbar und ermöglicht erst echte Verständigung, wogegen man ohne sie nur aneinander vorbeiredet." (ebd., S. 140, Hervorh. E. B.) „Gefühlsfreies Denken“ (ebd., S. 67) ist somit eine Illusion. Allerdings könne es zu einer „gleichmäßige[n] Gefühlsübereinstimmung“ im Rahmen einer „Kollektivstimmung“ kommen, die aus der Binnenperspektive eine „Gefühlsfreiheit“" suggeriert (ebd.). Damit ist gefühlsfreies Denken selbst ein stimmungsinduziertes Gefühl, das aus der ausgleichenden Energetik einer harmonischen Kollektivstimmung erwächst.

Besteht das Denkkollektiv über seine flüchtige, zufällige und kleine, d.h. dyadische Formation hinaus, wird es Fleck zufolge zum Träger eines spezifischen „Denkstils“. Voraussetzung dafür ist wiederum die Stimmung, die als Bereitschaft zur gerichteten Wahrnehmung die Möglichkeitsbedingung des Denkstils bildet. Sie schließt die ästhetische Dimension der sinnlichen Wahrnehmung, die kognitive Dimension des Sachlich-Gedanklichen und die soziale Dimension der praktisch vollzogenen Gerichtetheit zusammen. Denkstile zeichnen sich für Fleck zudem durch Regeln der Zusammenarbeit aus, sodass jenseits des additiven Zusammenfügens von Einzelleistungen ein organisiertes situatives Gebilde - etwa ein Orchesterkonzert oder ein

\footnotetext{
3 In seiner Definition des Denkkollektivs liefert Fleck eine Art Gründungsszene (vgl. Farzin und Laux 2014) dieses Theorems: „Ein Denkkollektiv ist immer dann vorhanden, wenn zwei oder mehrere Menschen Gedanken austauschen. Ein schlechter Beobachter, wer nicht bemerkt, wie ein anregendes Gespräch zweier Personen bald den Zustand herbeiführt, daß jede von ihnen Gedanken äußert, die sie allein oder in anderer Gesellschaft nicht zu produzieren imstande wäre. Eine besondere Stimmung stellt sich ein, der keiner der Teilnehmer sonst habhaft wird, die aber fast immer wiederkehrt, so oft beide Personen zusammenkommen. Längere Dauer dieses Zustandes erzeugt aus gemeinsamem Verständnis und gegenseitigen Mißverständnissen ein Denkgebilde, das keinem der Zwei angehört, aber durchaus nicht sinnlos ist. Wer ist sein Träger und Verfasser? Das kleine zweipersonale Kollektiv. Kommt ein Dritter hinzu, so macht er die frühere Stimmung verschwinden und mit ihr die besondere schöpferische Kraft des früheren Denkkollektivs; ein neues entsteht.“ (Fleck 1980 [1935], S. 60)
} 
Fußballspiel - entsteht. Diese meist ungeschriebenen Regeln lenken im Fall wissenschaftlicher Denkstile das Interesse auf bestimmte Probleme, sie legen evidente Urteile und erkenntnisfördernde Methoden fest. Hinzu kommt ein ,technischer und literarischer Stil des Wissenssystems" (ebd., S. 130), der sich in Fachsprachen und Jargons niederschlägt. Mit ihrer Stabilisierung durch die Bindung an ,organisierte soziale Gruppen“ erhalten Denkstile dann eine zunehmend gefestigte und ausgebaute „formale Struktur“ (ebd., S. 135), die durch eine „Hierarchie des Eingeweihtseins“ (ebd., S. 138) gekennzeichnet ist. Diese setzt einen inneren, ,esoterischen“ Kreis am Erkenntnisprozess aktiv teilnehmender Wissenschaftler:innen und einem äußeren, ,,exoterischen“ Kreis einer interessierten Öffentlichkeit voraus, deren Verhältnis Fleck mit den soziologischen Begriffen „Elite“ und „Masse“ charakterisiert.

Als Zugangsschwelle wird die kollektive Stimmung so zum Regulierungsinstrument der In- und Exklusion aus einem Denkkollektiv: Sie hält Andersdenkende fern. Denkkollektive zeichnen sich durch eine ,formelle und inhaltliche Abgeschlossenheit“" aus, deren Zugang durch Übergangsriten geregelt ist. Die Aneignung der denkstilspezifischen Regeln erfolgt im Rahmen einer „Lehrlingszeit, während welcher rein autoritäre Gedankensuggestion stattfindet, die nicht etwa durch ,allgemein rationellen " Gedankenaufbau ersetzt werden kann“ (ebd., S. 136). In dieser Formulierung wird deutlich, dass Denkkollektive immer auch „Suggestionskollektive“ sind - ein Begriff, den Fleck aus der parapsychologischen Forschung Karl Blachers zu okkulten Vereinigungen übernimmt (Bauer 2014, S. 88). Blacher hatte diesen Begriff im Anschluss nicht nur auf „Subjektkollektive“ wie Staat, Familie und Verein, sondern auch auf die Wissenschaft bezogen, um deren irrationale und affektive Dynamik hervorzuheben. ${ }^{4}$ Dieses Erbe klingt bei Fleck nach, wenn er den suggestiven Charakter mit der ,organische[n] Abgeschlossenheit jeder Denkgemeinde“ (Fleck 1980 [1935], S. 137) zusammenbringt. Um die innere Einheit zu stabilisieren, muss ein Denkkollektiv demnach eine deutliche Abgrenzung zu anderen Denkkollektiven vollziehen, deren Prinzipien, Fragen und Probleme als willkürlich, als mystisch, ja „als unwichtige oder sinnlose Spielerei“ (ebd., S. 143) abgetan werden. In dieser Weise entstehe eine ,spezifische Wertung und charakteristische Intoleranz", ja ein ,ausgesprochener Widerwille gegen denkstilfremdes Denken“ (ebd., S. 137). Eine solche „Denkstilbeharrung“ gipfelt darin, dass eine gewisse Distanz zweier Denkkollektive einen Austausch kategorisch unwahrscheinlich macht.

Mit Flecks Begriffsapparat des Denkkollektivs, des Denkstils und der Stimmung liegt ein vielversprechender Ausgangspunkt vor, um die atmosphärische Dimension von Theorien zu erfassen. Formale Aspekte wie Initiationsriten und Regeln geraten ebenso in den Blick wie die suggestive Wirkung etwa von Rhetoriken auf die Kollektivangehörigen und die Praktiken der Grenzziehung nach außen. Um Fleck aber für das Konzept von Theorieatmosphären nutzbar zu machen, muss seine Theorie von der massenpsychologischen Terminologie der 1930er-Jahre gelöst werden.

\footnotetext{
4 Wie Bauer aufzeigt, laufen in Flecks Denken wissenssoziologische Wurzeln im Anschluss an Wilhelm Jerusalem mit Bezügen aus der Umweltlehre Uexkülls und der Parapsychologie Blachers sowie Carl du Prels als historisches Apriori des Denkkollektivs zusammen. Gerade die Rolle Blachers ist hier bemerkenswert, da er neben frühen medienwissenschaftlichen Beobachtungen den ,latente[n] soziologische[n]“ Gehalt von Uexkülls Theorie der Umwelt affekttheoretisch weiterentwickelt (Bauer 2014, S. 96).
} 
Im Folgenden wird daher der Atmosphärenbegriff als geeignete Alternative zum Stimmungsbegriff umrissen. Der benachbarte Affektbegriff wird sich als hilfreich erweisen, um die Konzepte der Suggestion und des Gefühls einer Aktualisierung zu unterziehen.

\section{Atmosphären als Produkte affektiver Praxis}

Insofern er ausdrücklich auf den Raum zwischen Subjekt und Objekt abzielt, lässt der hier vertretene Atmosphärenbegriff Versuche der Lokalisierung von Atmosphären entweder in den Objekten oder im subjektiven Gefühl zunächst ins Leere laufen. Dennoch kann auch mit dieser Entscheidung nicht ignoriert werden, dass sich die Subjekt-Objekt-Dichotomie auf immer neue begriffliche Weise selbst in dezidiert relationale Konzeptionen einschreibt. Gerade für soziologische Atmosphärentheorien stellt das eine wiederkehrende Herausforderung dar.

Vertreter:innen der Neuen Phänomenologie wie Hermann Schmitz (2007, 2012) sind diesem Problem durch die Betonung der Leiblichkeit bzw. des ,eigenleiblichen Spürens" für die Entstehung von Atmosphären als „quasi objektiven Gefühlen“ begegnet (Schmitz 2007, S. 26). Im Anschluss daran hat Gugutzer eine neuphänomenologische Soziologie entworfen, die die ,affektive Betroffenheit“" sowohl als Apriori von Sozialität als auch als handlungstheoretisches Konzept versteht, das die antirationalen, antiteleologischen Bestandteile des Handelns hervorhebt (Gugutzer 2020, S. 188). Handlungsimpulse gehen aus dieser Sicht nicht von einem autonomen Subjekt, sondern von der ,affecting atmosphere“ einer Situation aus, die in ihrer Wirkung aber auf den leiblich spürenden Menschen zentriert bleibt. An diese leibphänomenologische Perspektive hat dann Gernot Böhme angeschlossen und zugleich Schmitz' „Ding-Ontologie“ kritisiert, die „Eigenschaften“ eines Dings wie Form und Farbe als dessen Bestimmungen fixiere (Böhme 1995, S. 32). Indem diese Bestimmungen als geschlossene, passive Größen gedacht würden, so Böhmes Kritik, werde ihr produktiver Einfluss bei der Herstellung von Atmosphären ausgeblendet. Auch wenn Böhme den transsubjektiven Charakter von Atmosphären mit dem Hinweis auf die „Ekstasen“ der Dinge hervorhebt, denkt er Atmosphären letztlich doch definitorisch als „Sphären gespürter leiblicher Anwesenheit“ (Böhme 2007, S. 37 f.). Bezugspunkt des Atmosphärenkonzepts bleibt also wiederum der subjektive Leib. ${ }^{5}$

Demgegenüber mehren sich in jüngerer Zeit Ansätze aus der kritischen Geographie, die, informiert durch die Affect Studies, die Räumlichkeit und Materialität von Atmosphären aus ihrer Fixierung in der leiblichen Präsenz menschlicher Subjekte zu lösen versuchen. Durch die Akzentuierung von Atmosphären als ,,affective atmospheres“ (Anderson 2009; Bissell 2010) wird der „elektrisierende“, Intensitäten erzeugende Charakter der Atmosphäre betont, ohne diesen auf Ausweichkategorien

\footnotetext{
5 Das gilt auch für Martina Löw, die auf die klassen-, geschlechts- und kulturspezifischen Aspekte von atmosphärischen Räumen verweist, deren differenzerzeugende Effekte sie in den Blick nimmt. Eine solche Konzeption bleibt ebenfalls der klassischen Gegenüberstellung von Subjekt und Objekt verhaftet, wenn die subjektive „Wahrnehmung“ anderer Menschen und ,sozialer Güter“ konzeptionell ins Zentrum gestellt wird (Löw 2001, S. 205 ff.).
} 
wie die persönliche Gestimmtheit, die Emotion oder das Gefühl, die allesamt auf die subjektive Wahrnehmung abheben, zu reduzieren. So konzipiert Derek McCormack (2008) Atmosphären als zugleich meteorologische und affektive Räume, Nigel Thrift spricht von „mimetic waves“ (2008), Bisell von „modes of affective transmission“ (2010). Neben der Räumlichkeit rückt dabei auch die zeitliche Dimension von Atmosphären zunehmend in den Fokus: Atmosphären sind demnach keine in sich geschlossenen statischen Räume, sondern durch temporale Register und Mikrotemporalitäten, d.h. Be- und Entschleunigungen, Rhythmen und Wiederholungen, plötzliche Richtungswechsel und Stagnationen gekennzeichnet (Salter 2014). In der wiederkehrenden Wendung der ,affektiven Atmosphären“, die sich ebenso durch die neuphänomenologischen wie durch die affekttheoretischen Beiträge zieht, wird oftmals eine enge Kopplung von Affekt und Atmosphäre unterstellt, ohne dass deren Verhältnis genauer geklärt würde. ${ }^{6}$

Mit Blick auf diese Gleichsetzung fällt in der Diskussion eine Spannung auf: Während der Atmosphärenbegriff u.a. aufgrund seiner Herkunft aus der Physik als zu starr kritisiert wird (Wagner 2007), gilt der Affektbegriff aus soziologischer Perspektive häufig als vage und romantisierend (Wetherell 2013). Zur Abgrenzung beider Begriffe hält Friedlind Riedel fest: ,While , affect‘ refers to the ways in which (emerging) bodies relate to each other [...], , atmosphere' describes the ways in which a multiplicity of bodies is part of, and entrenched in, a situation that encompasses it." (Riedel 2019, S. 85). Das Konzept der Atmosphäre abstrahiert also zunächst von den Körpern und Dingen, die von ihr umgeben sind, und zielt auf die situativen Wirkungen, die das räumliche „Dazwischen“ als Ganzes auf seine einzelnen Bestandteile ausübt. Diese Generalisierung, die eine einheitliche Wirkung auf alle der Atmosphäre ausgesetzten Körper unterstellt, kann dann entweder wie bei Martina Löw (2001) zum Einsatzpunkt soziologischer Anpassungen des Atmosphärenbegriffs werden - oder aber zum Anlass, den Atmosphärenbegriff mit Verweis auf dessen deterministische Implikationen gänzlich fallen zu lassen (Seyfert 2012).

Möglicherweise ist es eine Reaktion auf diese konzeptionelle Sackgasse, dass innerhalb der Atmosphärendiskussion die Rufe lauter werden, an konkreten empirischen Fällen zu zeigen, wie Atmosphären in je spezifischen Feldern auf die

\footnotetext{
${ }^{6}$ Es mag der Vagheit des Affektbegriffs geschuldet sein, dass er oftmals in die Nähe des nicht weniger vagen Atmosphärenbegriffs gerückt wird, sodass beide Konzepte in ihrer Ungenauigkeit nahezu tautologisch aufeinander zu verweisen scheinen (vgl. Bille et al. 2015). Die Annahme, dass Atmosphären per definitionem affektiv funktionieren und umgekehrt, würde in der Konsequenz die Rede von ,,affective atmospheres“ bzw. den ,,atmospheres of affect“ (Salter 2014) hinfällig machen. Deutlich wird diese Tendenz zum Tautologischen etwa bei Brian Massumi, der den Begriff der ,,affective atmosphere“ synonym mit seinem Zentralbegriff der ,affective tonality“ verwendet (Massumi 2011, S. 112). Letztere wird gefasst als „embracing atmosphere that is also at the very heart of what happens because it qualifies the overall feel. Affective tonality is what we normally call a ,mood““ (ebd., S. 65). Stimmung, Atmosphäre und Affekt werden also im Begriff der ,,affective tonality“ unvermittelt kurzgeschlossen. Wie Jan Slaby in seiner Rekonstruktion von „drei Haltungen der Affekttheorie“ anmerkt, handelt es sich bei Massumis „Schreib- und Denkstil“ um ,eine auf rhetorische und dynamische Wirksamkeit abzielende Begriffs- und Theoriepoetik, die eine performative und ästhetische Entsprechung zum inhaltlich Mitgeteilten anstrebt“ (Slaby 2018, S. $61 \mathrm{f}$.). Der Umstand, dass die Texte Massumis selbst hochgradig atmosphärisch funktionieren, scheint dabei den Ruf nach begrifflicher Präzision geradezu aufzuheben.
} 
unterschiedlichen involvierten Akteure wirken. ${ }^{7}$ So ist Margaret Wetherells Konzept der ,,affective practice“, das den Affektbegriff praxistheoretisch unterfüttert, auf empirische Untersuchungen zu Atmosphären zugeschnitten. Das Konzept macht sich zur Aufgabe, einerseits die in der klassischen soziologischen Emotionsforschung angenommene soziale Erlern-, Form- und Kontrollierbarkeit von Gefühlen und andererseits die in der Affekttheorie betonten Aspekte der Emergenz, des UnerwartetÜberraschenden und des Kontrollverlusts zusammenzubringen (Wetherell 2013, S. $221 \mathrm{ff}$.). ${ }^{8}$ Damit fungiert der Begriff der ,affektiven Praxis“ auch als Korrektiv zur Tendenz innerhalb der Affekttheorie, Affekte als ,unspecified force, unmediated by human consciousness, discourse, representation and interpretation“ zu verstehen (ebd., S. 228).

Wetherell kritisiert die Dezentrierung des Subjekts und die Ausblendung von Diskursen innerhalb der non-repräsentationalen Affekt- und Atmosphärendiskussion, ohne auf deren analytische Zugewinne - insbesondere die Rolle irrationaler, irregulärer und unerwarteter Momente in Interaktionsprozessen - verzichten zu wollen. Auf diese Weise kann sie untersuchen, wie affektive Atmosphären einerseits auf die Formation von Subjektivität sowie auf konkrete Subjekte einwirken, und wie andererseits die ,affizierten Subjektivitäten“ umgekehrt aktiv Atmosphären gestalten, verändern und neu hervorbringen. Dieser reflexive und empirisch fruchtbare soziologische Anschluss an die Affekttheorie erlaubt es, Wetherells Konzept der affektiven Praxis der folgenden Analyse von Theorieatmosphären zugrundezulegen. Indem wir die „Arbeit“" von Denkkollektiven als affektive Praxis im Sinne Wetherells begreifen, werden sowohl ihre formale Struktur wie ihre Exzesse, ihre Beharrlichkeit wie ihre Wanderbewegungen, ihre Berechenbarkeit und ihre unerwarteten Wirkungen sichtbar.

Aktualisieren wir nun Flecks Theorie der Denkkollektive mithilfe der Erträge der neueren Affekttheorie, dann beschränkt sich der Kollektivbegriff nicht länger auf menschliche Kollektive, sondern umfasst auch Objekte und materielle Artefakte, die zu aktiv Teilnehmenden bei der Erzeugung affektiver Atmosphären werden. Auch wenn Fleck den Begriff der Stimmung nicht als subjektive Emotion, sondern ganz im Sinne des Atmosphärenkonzepts als Zwischenraum begreift, bleibt der Begriff als vom menschlichen Kollektiv ausgehende Energie gedacht. Der allgemeinere Begriff der Atmosphäre aber ist geeignet, die im Stimmungsbegriff unterbelichtete Rolle von Dingen, Räumen und Technologien in ihrem produktiven Einfluss auf ,,affektive Arrangements“ (Slaby 2019) zu berücksichtigen. So wird verständlich, dass Denkkollektive nicht zuletzt vermittels ihrer Dingkultur eigentümliche ästhetische und affektive Überschüsse produzieren, die das Denken auf nichtintendierte Weise strukturieren.

\footnotetext{
7 Dass es nicht bei Rufen geblieben ist, zeigen u.a. ethnografische Forschungen zu den affektiven Atmosphären urbaner Räume (Thibaud 2011), öffentlicher Sportveranstaltungen (Edensor 2015), von Transportinfrastrukturen (Bissell 2010) und forensischen psychiatrischen Einrichtungen (Brown et al. 2019).

8 In Slabys Kartierung des Feldes der Affekttheorie kommt Wetherell die „Haltung“ der Forscherin zu (Slaby 2018 65ff.), da sie Emotion und Affekt einer systematischen sozialwissenschaftlichen Erforschung zugänglich macht und sich dazu auch vom idiosynkratischen Jargon der philosophisch-kulturwissenschaftlichen Affekttheorie à la Massumi sowie der aktivistischen Haltung einer Sarah Ahmed abgrenzt.
} 
Für die Analyse können aus der bisherigen Diskussion drei Charakteristika von „Atmosphären“ festgehalten werden. So überschreiten und verwirren Atmosphären erstens einfache Gegenüberstellungen von Subjekt und Objekt, Innen und Außen, Diskurs und Materialität und machen stattdessen das „Dazwischen“ als einen Raum der Vermittlung und Vermischung denkbar. Zweitens müssen Atmosphären als ein situationales raumzeitliches Phänomen gelten: Sie sind das Produkt der heterogenen mehr-als-menschlichen Körper, welche sie räumlich umgeben, und überschreiten immer schon den individuellen Körper. In diesem Sinne sind Atmosphären der relationale und emergente Effekt eines Gefüges, dessen Muster von Flüchtigkeit und Stabilisierung, von Ansteckung und Begrenzung erst im zeitlichen Verlauf hervortreten. Atmosphären funktionieren drittens affektiv in dem Sinne, dass sie auf primär nichtbewusste, nichtrationale und nichtintentionale Weise auf das Vermögen von Körpern einwirken. Dabei handelt es sich nicht um ein passives Empfangen. Atmosphären sind vielmehr selbst als affektive Praxis zu verstehen; von Interesse ist nun das ,,doing atmosphere“.

\section{Theorieatmosphären}

Während ein Großteil der einschlägigen Atmosphärenstudien auf Face-to-face-Situationen in physischer Kopräsenz abstellt, werden Theorieatmosphären vor allem durch den Text, Objekte und Räume erfahrbar. Bei ihrer Analyse wird es darauf ankommen, die Praktiken der Konstruktion, Rezeption und Inszenierung von Theorie nicht als getrennte Bereiche zu betrachten, sondern das jeweils vermittelnde Dazwischen freizulegen. Der Fokus auf das relationale Moment der Atmosphäre soll es ermöglichen, die Affektivität von Theorie in den Blickpunkt zu rücken - und damit ein Moment von Theorie, das dem mit ihr gemeinhin verknüpften Wert der Rationalität entgegensteht. Daher interessieren gerade auch die nichtintendierten Wirkungen von Theorie jenseits reflexiver Konstruktionen, Absichten und Strategien.

Exemplarischer Gegenstand der folgenden Ausführungen sind der luhmannianische und der deleuzianische Denkstil, und damit zwei der erfolgreichsten Paradigmen der jüngeren soziologischen Theorie. Weil es sich hier nicht nur um konträre, sondern auch um konkurrierende Denkstile handelt, deren Denkkollektive sich jeweils durch eine starke esoterische Beharrungstendenz auszeichnen, lassen sich im Vergleich sowohl Grenzkämpfe als auch die Gemeinsamkeiten jener affektiven Praktiken herausarbeiten, die die Formierung der jeweiligen Theorieatmosphäre ermöglichen. Als historischer Referenzpunkt dient die Hochzeit der westdeutschen Theoriebegeisterung in den 1970er- und 1980er-Jahren, da hier, wie von Felsch (2015) gezeigt, die affektiven Wirkungen der Theorieatmosphären besonders deutlich hervortreten. „Theorie“ wurde in der damaligen Konstellation selbst zu einem hochgradig schillernden atmosphärischen Begriff, der in dieser emphatischen Aufladung neben der Kritischen Theorie vor allem die Systemtheorie Luhmanns und die poststrukturalistischen Theorieansätze u.a. von Deleuze und Guattari umfasste.

Die Auswertung des Materials erfolgt anhand von drei Dimensionen von Theorie, die sich über ihre atmosphärische Wirkung entfalten. Erstens wird die Ebene des theoretischen Textes in den Blick genommen, auf der sich textliche und paratextli- 
che Komponenten, d.h. Techniken, Materialien und Methoden der Begriffsbildung, der Systematisierung und Plausibilisierung zu einem denkstilspezifischen „Theoriesound“"verdichten. Daran anschließend geht es zweitens um die Lesepraktiken des exoterischen Denkkollektivs, das in Relation zum Text eine bestimmte Rezeptionsatmosphäre erzeugt. Drittens werden die Dinge und Räume der Theorie fokussiert, was sowohl Infrastrukturen, Objekte und Artefakte eines Denkstils als auch die außertextlichen Inszenierungsweisen umfasst, die zu einer affektiven Zirkulation der Theorie beitragen. Wenn zunächst an der konventionellen Gliederung dieser drei Dimensionen festgehalten wird, so handelt es sich um eine in erster Linie analytische Trennung, die den Blick nach und nach auf die Zwischenräume freilegen soll, in denen eine je spezifische Theorieatmosphäre entsteht. ${ }^{9}$

\subsection{Der Text}

An der Entstehung einer Theorieatmosphäre ist zunächst wesentlich die Textoberfläche beteiligt: Ein Text liest sich als Sound und als Rhythmus. Er besteht aus unterschiedlich langen Sätzen, aus Verdichtungen und Reduktionen, aus Übergängen und Brüchen. Die Wirkung eines Textes speist sich zum anderen aus kompositorischen Prinzipien des Denkstils, d.h. aus der Theoriearchitektur, aus dem Abstraktionsund Komplexitätsgrad der Begriffsbildung, die in einem Kollektiv als akzeptiert gelten. Diese formalen Momente verschränken sich konstitutiv mit der Sachdimension, können aber mitunter eine sich verselbstständigende - und der Sachdimension widerstrebende - Eigendynamik freisetzen. Die ganz unterschiedlichen Metaphern des Sounds, der heißen und kalten, hellen und dunklen, schnellen und langsamen Effekte von Theorie erweisen sich als geeignete begriffliche Mittel, um ausgehend von den Selbstbeschreibungen aus dem Material die Funktionsweise von Theorieatmosphären zu erfassen.

\subsubsection{Die eisigen Höhen der Abstraktion: Der systemtheoretische Denkstil}

Wenn Luhmann gelegentlich für ein moralisches ,supercooling“"10 der theoretischen Analyse eintritt, dann tut er das auch vermittels eines nüchtern-abstrakten ,Sounds“ und eines komplexen, logisch-stringenten, in sich geschlossenen Denkstils, der zugleich das Spiel mit Paradoxien zum Programm macht. An die Stelle eines Theorieentwurfs, der ,schäumt und ohne klaren Duktus Komplexität ansammelt“, tritt eine Theorie selbstreferentieller, autopoietischer Systeme, die sich durch einen „sehr viel höheren, sich selbst reflektierenden Grad begrifflicher Komplexität“" (Luhmann 1984, S. 11) auszeichnet. Mangels soziologischer Vorbilder muss sie auf die denkstilfremden Begriffs- und Ideenapparate der Zellbiologie, der Physiologie sowie der

\footnotetext{
9 Das von Philipp Felsch präsentierte Daten- und Archivmaterial ist für die folgende Darstellung maßgeblich. Dieser empirischen Ausgangslage ist es geschuldet, dass der Ebene des Textes vergleichsweise große Aufmerksamkeit zukommt, wobei diese programmatisch um die beiden anderen Dimensionen der Rezeption und der Dinge erweitert wird. Die historische Distanz zu diesem hochgradig affektiven Umgang mit Theorie erlaubt überdies, am Schluss einige Schlaglichter auf die Historizität von Theorieatmosphären und ihre Abhängigkeit von größeren gesellschaftlichen Atmosphären zu werfen.

${ }^{10}$ Luhmann zitiert nach Rasch und Knodt 1994, S. 6.
} 
Computerwissenschaft, hier vor allem Kybernetik, Steuerungs- und Automatentheorie, zurückgreifen. Um dem Denkstil Kontur zu verleihen, vollzieht Luhmann eine innovative atmosphärische Öffnung für fachfremde Denkstile. Diese Vorgehensweise bringt einen innerhalb der Soziologie neuartigen Theoriesound hervor. Statt wie gängige soziologische Theorieverfahren direkt an vorhandene Begriffstraditionen anzuknüpfen, solle, so Luhmann in der Einleitung von Soziale Systeme,

im folgenden versucht werden, die Zahl der benutzten Begriffe zu erhöhen und sie mit Bezug aufeinander [Hervorh. i. Orig.] zu bestimmen. Das geschieht mit Begriffen wie: Sinn, Zeit, Ereignis, Element, Relation, Komplexität, Kontingenz, Handlung, Kommunikation, System, Umwelt, Welt, Erwartung, Struktur, Prozeß, Selbstreferenz, Geschlossenheit, Selbstorganisation, Autopoiesis, Individualität, Beobachtung, Selbstbeobachtung, Beschreibung, Selbstbeschreibung, Einheit, Reflexion, Differenz, Information, Interpenetration, Interaktion, Gesellschaft, Widerspruch, Konflikt. (ebd., S. 12)

Diese Liste von Begriffen ist in ihrer modularen Form der Aufzählung charakteristisch für einen Stil des Theoretisierens, dessen Hauptaufgabe im Arrangieren und Anordnen dieser Baukasten-Elemente im Rahmen einer geschlossenen, ,selbsttragende[n] Konstruktion“ (ebd., S. 11) besteht. Dieses architekturhafte „Bauen“ von Theorie beruht aber ebenso auf einem Stil im ästhetischen Sinne, der sich als modularer Sound in den Text einschreibt - und der paradoxerweise klingt, wie er klingt, weil sich sein Autor nicht als Stilist im eigentlichen Sinne begreift. Wenn Luhmann bemerkt, „Theorieform und Darstellungsform“ (ebd., S. 14) nicht in Einklang bringen zu wollen, dann meint er damit die zwar in der Schlussfassung logisch aufeinander aufbauende, an sich aber arbiträre architektonische Form der Kapitelfolge in Soziale Systeme. Im Sinne des modularen Baukastens erscheint die Darstellungsform allein als eine Frage der „Arrangierprobleme“. Abgesehen davon handele es sich um eine Theorie, die, ,was die Begriffsfassungen und die Aussagen inhaltlich angeht, sich wie von selbst geschrieben hat" (ebd.).

Wie klingt eine solche Theorie? Die naheliegende Antwort wäre, dass sie flächigabstrakt klingt, weil Abstraktion eine ,erkenntnistheoretische Notwendigkeit“" (ebd., S. 13) darstellt. Der Sound wäre in dem Sinne ein reines Nebenprodukt theoretischer Zwänge, unbedeutend für den Prozess des Theoretisierens. Um Erfordernis und Charakter der angestrebten theoretischen Abstraktion zu verdeutlichen, bedient sich Luhmann einer so vielschichtigen wie vielsagenden Metapher:

Diese Theorieanlage erzwingt eine Darstellung in ungewöhnlicher Abstraktionslage. Der Flug muß über den Wolken stattfinden, und es ist mit einer ziemlich geschlossenen Wolkendecke zu rechnen. Man muß sich auf die eigenen Instrumente verlassen. Gelegentlich sind Durchblicke nach unten möglich ein Blick auf Gelände mit Wegen, Siedlungen, Flüssen oder Küstenstreifen, die an Vertrautes erinnern; oder auch ein Blick auf ein größeres Stück Landschaft mit den erloschenen Vulkanen des Marxismus. Aber niemand sollte der Illusion zum Opfer fallen, daß diese wenigen Anhaltspunkte genügen, um den Flug zu steuern. (ebd., S. 12f.) 
Das atmosphärisch-meteorologische Wolkenbild hat hier mehrere Funktionen: Es macht deutlich, dass die Frage des Arrangements nicht nur auf die Anordnung eigener begrifflicher Mittel beschränkt ist, auf die es sich zu verlassen gilt, sondern auch der Selbstpositionierung im Feld anderer Theorien und innerhalb der sozialen Wirklichkeit dient. Es ist eine „kühle“ Beobachterposition, die aus großer Entfernung auf die überhitzten ,Vulkan“-Theorien blickt, deren „Erloschenheit“ sie als Ruinen der Vergangenheit besiegelt.

Obwohl Luhmann hier den ,heißen“ Theorien keine Orientierungsfunktion für das eigene Theoretisieren zugesteht, erfüllt das Atmosphärenbild genau diese Funktion: Erst durch die Bestimmung des Abstands zu ihnen wird die eigene Position konturiert. Die Abstraktion ist aus dieser Perspektive nicht einfach Sachzwang einer Theorie, die sich von selbst schreibt, sondern sie hat selbst in doppelter Hinsicht einen atmosphärischen Charakter: erstens, insofern sie einen Sound produziert; zweitens, insofern sie darauf angewiesen ist, Ortsbestimmungen, Abstände und Grenzziehungen im Gefüge konkurrierender Denkstile vornehmen zu müssen. Das atmosphärische Kältebild scheint bei Luhmann fast für solche trennenden - und darin wieder verbindenden - Grenzziehungen reserviert zu sein. Luhmanns moralisches ,,supercooling“ ist so eingängig, weil es als Provokationsmittel gegenüber dem Selbstverständnis der Soziologie als warmer, d.h. kritischer, engagierter, mitfühlender Wissenschaft angelegt ist. ${ }^{11}$ Auf diese Denkstilbeharrung der Systemtheorie wird später im Kontext der Luhmann-Habermas-Debatte noch einmal zurückzukommen sein.

\subsubsection{Die feuchtwarmen Gebiete des Rhizoms: Der deleuzianische Denkstil}

Die französische Tradition des Poststrukturalismus und der Dekonstruktion kann neben der Kritischen Theorie Frankfurter Provenienz als die vielleicht größte Konkurrentin des luhmannianischen Denkstils in den Sozialwissenschaften gelten. Auch wenn die Unterschiede zwischen den Autor:innen dieser Tradition nicht zu unterschätzen sind, zeichnen sich diverse unter ihnen durch einen bewusst konträren, „heißen“ Theoriesound aus. So wird jener Distanz zwischen Theorie, Politik und Sinnlichkeit, wie sie Luhmann vorschwebte, von Gilles Deleuze und Félix Guattari ein ,überhitzter“, ja überschäumender Denkstil des endlosen Werdens und rhizomatischen Wucherns entgegengestellt. ${ }^{12}$

\footnotetext{
11 Im Wintersemester 1968/69, also auf dem Höhepunkt studentischer Kämpfe, vertrat Niklas Luhmann in deren Epizentrum Frankfurt am Main die Professur Theodor W. Adornos. Dem heutigen Kenntnisstand zufolge bot er dort eine Übung an zur Soziologie der Liebe (Anz 2010). Das überlieferte Diskussionspapier (Luhmann 2008) enthält bereits den Grundgedanken des späteren Klassikers Liebe als Passion (Luhmann 1982): Aus soziologischer Perspektive ist die amouröse Leidenschaft kein „Gefühl“, sondern ein „,Kommunikationsmedium“. Ein stärkerer Kontrast zum „heißen“ Zeitgeist von 1968 wäre schwer auszudenken.

12 Wenn von „heißen“ und „kalten“ Theorien die Rede ist, liegt McLuhans Begrifflichkeit heißer und kalter Medien nahe. McLuhans Verständnis macht sich an der Partizipation fest, die ein Medium vom Rezipienten verlangt: Heiße Medien verlangen von der Nutzerin einen eher geringen Grad der Partizipation, während kalte Medien intensive Beteiligung erfordern (McLuhan 1964, S. 24f.). Im Sinne der bisherigen Analyse des Theoriesounds fällt die Codierung der heiß/kalt-Unterscheidung kontraintuitiv aus. Heiß wären demnach Theorien, die durch ihre logische Geschlossenheit ein geringes Maß an Ergänzungsund Vervollständigungsarbeit voraussetzen; kalt wären solche, die ihr Publikum durch Auslassungen, An-
} 
Als dessen Medium fungiert eine bestimmte Buchform, die zu ihrer Konturierung wiederum einer Negativfolie bedarf: In Abgrenzung vom in der Struktur baumhaften, sich als Ausdruck ,schöne[r] Innerlichkeit, organisch, signifikant und subjektiv“ präsentierenden konventionellen Buch (Deleuze und Guattari 1992, S. 14) entwerfen Deleuze und Guattari ihre Tausend Plateaus als antigenealogisches, mannigfaltiges Buch, das keine „Punkte“ oder „Positionen“, sondern nur noch „Linien“ kennt (ebd., S. 18). Statt von Kapiteln, die „Höhe- und Schlusspunkte“ des Buches markieren, sprechen die Autoren von gleichmäßigen „Plateaus“ (ebd., S. 37), die von jeder beliebigen Stelle aus gelesen und miteinander verknüpft werden können. Dieses Formprinzip, das auf dem Cover der 1992 im Merve-Verlag erschienenen deutschen Übersetzung des 1980 erschienenen Originals und im Inhaltsverzeichnis visualisiert ist, erinnert auf den ersten Blick an Luhmanns modularen Theorieaufbau. Durch den ,heißen Sound“, der bei Deleuze und Guattari mit dieser Form zusammenfällt, entfaltet sich aber eine geradezu konträre Theorieatmosphäre.

Das zeigt sich schon mit Blick auf die paratextlichen Elemente. Diese Elemente, die im Sinne des Literaturwissenschaftlers Gérard Genette (1989) zum Buch, nicht aber zum Werk gehören, sind ein wichtiger Teil der Atmosphärenwirkung. Sie umfassen den oder die Autor:innennamen, den Buchtitel, das Inhaltsverzeichnis, vorangestellte Widmungen und Motti, das Vorwort, Zwischentitel, Anmerkungen der Herausgeber:innen und vieles mehr. Wie Genette schreibt, haben diese Elemente die Funktion, den Text ,zu präsentieren: ihn präsent zu machen, und damit seine ,Rezeption “ und seinen Konsum in, zumindest heutzutage, der Gestalt eines Buches zu ermöglichen“ (ebd., S. 9). Der Paratext ist das Beiwerk, durch das ein Text zum Buch wird und als solches vor die Leser:in tritt. Ob ein Buch gelesen wird oder nicht, entscheidet sich oftmals an seinem Titel, d.h. daran, ob dieser spannend klingt, eine Atmosphäre der Spannung, gar des Geheimen erzeugt, das es zu ergründen gilt. Ganz im Sinne des Atmosphärenkonzepts formen und steuern die paratextlichen Elemente zwar die Lektüre und sind damit hochgradig präsent, bleiben als Bestandteile, Rahmungen und Motoren der Rezeption meist aber weitgehend unsichtbar.

Auf Tausend Plateaus bezogen, wird das mit dem Cover und Inhaltsverzeichnis angekündigte „Wuchern“ in Begriffsbildung und Theoriesound aufgenommen und weitergeführt. In ihrem assoziativen, ja halluzinatorischen Ton verfremden Deleuze und Guattari die denkstilspezifische Rhetorik der Wissenschaft insgesamt. Wüste Aufzählungen, Exklamationen, Fäkalsprache, ein Sound der Beschleunigung und Überhitzung durchziehen das Buch:

Auch das antikulturelle Buch kann mit einer schweren kulturellen Last beladen sein, doch es wird sie aktiv benutzen: Vergessen statt Erinnerung, Unterentwicklung statt Fortschritt zur Entwicklung, Nomadentum statt Seßhaftigkeit, Karte statt Kopie. RHIZOMATIK = POP-ANALYSE [...]. (Deleuze und Guattari 1992, S. 40)

deutungen und Sprünge konstant zum Hinzudichten zwingen (vgl. dazu auch Galloway 2018). Luhmann wäre also plötzlich „heiß“, Deleuze „,kalt“. Um Verwirrung zu vermeiden, wird an dieser Stelle auf die McLuhan'sche Begriffsverwendung verzichtet, ohne dabei die Dimension der Partizipationsarbeit aus dem Blick zu verlieren. 
Anders als Luhmanns „Cooling“-Strategien stellt dieser exzessive Sound seinen Atmosphärencharakter offen aus. Dass das in den 1970ern eine heftige Provokation war, zeigt sich nicht zuletzt daran, dass Deleuze und Guattari wie auch dem Poststrukturalismus insgesamt der Theoriestatus innerhalb des fachübergreifenden Denkstils der Soziologie lange Zeit schlicht abgesprochen wurde. Inzwischen irritiert die Feier des Horizontalen und Nomadischen weniger - was nicht nur daran liegt, dass deleuzianische Denkkollektiv sie längst zu einem kanonisierten Denkstil fortentwickelt hat, sondern auch an ihrem Erfolg außerhalb der Grenzen der Theorie. Bekanntlich haben auch New-Economy-Ideologen den Deleuze'schen Sound für sich entdeckt, wie Alexander Galloway beobachtet:

[W]e must forget the Google Deleuzians, those who see the world as a vital assemblage, proffering untold bounties of knowledge - and riches [...]. Systems are open, dynamic, and robust. Networks produce value. These are some of the many mantras of the Google Deleuzians. (Galloway 2015)

Dass die deleuzianische Theorieatmosphäre - vermittelt über Mantren als rituelle Mittel - in „feindliches Gebiet“ eingewandert ist, verändert indes die Bedingungen ihres Wirkens. Diese Entwicklung nimmt auch Andrew Culp in seinem Buch Dark Deleuze (2015) zum Anlass, in der schwärmerischen, ja hippieesken Feier der exzessiven Verbindungen eine andere, verschüttete, düstere Linie bei Deleuze freizulegen:

The most immediate instance of lightness, connectivism, is the realization of the techno-affirmationist dream of complete transparency [...]. Such an untimely descent into darkness begins with a protest: lightness has far too long been the dominant model of thought. The road there descends from the chapel to the crypt. (Culp 2015)

Zur dunklen „Krypta“ arbeitet sich Culp vor, indem er der Deleuze'schen „Assemblage“ das „Nicht-Werden“ entgegensetzt, der „Intensität“ die „Grausamkeit“, der Affirmation den Pessimismus gegenüber der bestehenden Welt.

Galloway und Culp sind Beispiele für den esoterischen Kreis des deleuzianischen Denkstils, den sie wider der rigorosen Beharrungs- und Schließungstendenz von Denkstilen einer Selbstkritik unterziehen, um weiter an ihm festhalten zu können. Bei Culp geschieht dies auf atmosphärischem Wege: Eine „dunklere“ Theorieatmosphäre soll das Vokabular der Kritik retten. Der Theoriesound macht sich also nicht nur an der Unterscheidung von ,heiß“ und „kalt“ fest, denn hier taucht mit „hell“ und „dunkel“ eine andere atmosphärische Begrifflichkeit auf. Diese beiden Gegenüberstellungen sind nicht einfach deckungsgleich: Culps Text ist nicht „kühl“, sondern selbst in einer überhitzten Manifestform verfasst.

Denkbar wären viele weitere Akzentuierungen: Die temporale Struktur eines Denkstils mag als „schnell“ oder „langsam“ erscheinen, ein tastendes Vorgehen als „leise“ und „weich“, ein vorpreschendes als ,laut“" und „hart“. Die diskutierten Denkstile sind also nicht an sich ,heiß“ oder ,kalt“, „,hell“ oder ,dunkel“, sondern werden es erst in Relation zu konkurrierenden Denkstilen und den damit verbundenen Theorieatmosphären. Die vielfältigen Interventionsgesten führen vor, dass die textlich transportierten Komponenten der Atmosphäre nicht in sich geschlos- 
sen funktionieren, sondern immer schon in Umgebungen der Rezeption und der Schulbildung sowie in theoretische Deutungskämpfe eingelassen sind, deren historische Gebundenheit das Denkkollektiv auch über die Referenzautor:innen hinaus zu Weiterentwicklungen und Anpassungen zwingt. Immer wieder hat sich außerdem gezeigt, dass die Theorieatmosphäre ihr angestammtes Feld der Theorie verlässt und über den Text hinaus in andere Bereiche vordringt: in die Ökonomie, in die Literatur, in die Popkultur, in den Journalismus. Nicht zuletzt das deutsche Feuilleton zeigt sich bei Rezensionen hochempfänglich für Theorieatmosphären. Diese Ausbreitung zeugt einerseits vom Erfolg einer Theorieatmosphäre, verändert aber umgekehrt ihre Wirkung, was Neupositionierungen und Verschiebungen, neue „Abkühlungen“ oder „Erhitzungen“ notwendig macht.

\subsection{Die Praxis des Lesens}

In seiner Analyse der „Haltungen“ innerhalb der Affekttheorien hält Slaby mit Blick auf die Rolle atmosphärischer Momente in der Theorierezeption fest:

Akademische Texte transportieren nicht nur semantische Gehalte, sondern erzeugen einen affektiven Resonanzraum, der Leser/innen zu unterschiedlichen Formen des Mit- oder Gegenschwingens animieren und sie auf diese Weise motivieren, begeistern, aber auch abstoßen kann. (Slaby 2019, S. 56)

Während Slaby diese affektive Dimension des Lesens als „Ergebnis einer bewusst praktizierten Strategie der jeweiligen Verfasser/innen“ (ebd.) deutet, dient der hier vorgeschlagene Atmosphärenbegriff im Gegenteil dazu, die souveräne Autorenfigur zu relativieren. Autor:innen mögen ihre Texte in gewisser Absicht gestalten; aus der Perspektive Flecks sind sie jedoch in ihrer Auswahl von Gegenständen, aber auch in ihrem Denkstil wesentlich von den Denkkollektiven ihrer akademischen Heimat und Umgebung abhängig. Auch der Sound einer Theorie, der auf den ersten Blick im Text selbst zu verorten ist, entfaltet seine atmosphärische Wirkung erst in Beziehung zur Rezeptionsarbeit des esoterischen wie exoterischen Denkkollektivs, wobei Paratexte und Buchcover das Interface zwischen Text und Leser:in bilden.

Eine Theorieatmosphäre geht also nicht einfach vom Text aus, sondern beginnt erst durch das Denkkollektiv und seine affektive Praxis des Lesens Kontur zu erhalten (Engert und Krey 2013). So setzt der Theoriesound eine Leser:in voraus, die sich in der Gebrauchspraxis von ihm affizieren lässt. Laut Fleck erwecken ,gute, stilvolle Arbeiten [...] sofort entsprechende solidarische Stimmung beim Leser, und sie ist es, die nach einigen Sätzen das Buch zu schätzen zwingt und wirkungsvoll macht“ (Fleck 1980 [1935], S. 189). Es ist also eine suggestive Sogwirkung, die ein bestimmter Stil unabhängig vom Mitgeteilten zu entfalten beginnt. Die Leser:in erscheint bei Fleck indes eher als passive Empfänger:in des atmosphärischen Zwangs. Um solche mechanistischen Untertöne zu vermeiden, ist es vielversprechender, vom Lesen als affektiver Praxis auszugehen, in deren Rahmen der Text nicht nur kognitiv angeeignet wird. Das entspricht McLuhans Beobachtung, dass Textrezeption sich nie nur hermeneutisch, sondern immer auch sinnlich vollzieht. Denn offenkundig ist beim Lesen von Theorie das logische Verstehen untrennbar verbunden mit affektiven Mustern, die von Vergnügen über Ehrfurcht und Anstrengung bis hin zu Angst und 
Einschüchterung reichen und die Geschwindigkeit und Reihenfolge einer Lektüre steuern.

Als Kronzeuge dieser Beobachtung kann Rainald Goetz gelten, in dem Luhmann einen prominenten Leser findet, der in seinen Leseberichten immer wieder die Affektivität der Verstehensdimension selbst thematisiert. ${ }^{13}$ So begründet Goetz seine Faszination für Luhmann im Roman Abfall für Alle:

Mich ERSCHÜTTERT Luhmanns Totale, immer wieder, und zwar weil ich finde, daß sie selbst so erschüttert ist. [...] Bloß hat sich Luhmann angenehmerweise nie dafür interessiert, aus dem seinem Denken zugrunde liegenden existenziellen Beben eine Nummer zu machen, einen Auftritt [...]. (Goetz 1999, S. 160)

Die Spannung in Luhmanns Denkstil, der seinen Stilgehalt performativ zurückweist, entfaltet erst in der affektiven Praxis des Lesens ihre paradoxe Wirkung: Goetz formuliert ein ,heißes“ Lob für Luhmanns „Kälte“. Während das hypersachliche Selbstverständnis Luhmanns von Soziolog:innen in der Regel für bare Münze genommen wird, veranlasst der atmosphärische Reiz dieser Sachlichkeit exoterische Teilnehmer des Denkkollektivs wie Goetz dazu, die Systemtheorie in einem systemfremden, nämlich ästhetischen Vokabular als ,ultimatives Kunstwerk“ zu beschreiben (vgl. Werber 2000). Hinzuzufügen wäre: Kunstwerk nicht im klassischen Sinn. Was den Sound betrifft, ist Luhmann lesen Goetz zufolge eher mit Techno hören zu vergleichen. In seinem Roman Rave demonstriert Goetz (2001), wie beide Sounds - der von Luhmann und der von Techno - in ihrem repetitiv-modularen, knappen, kühlen Charakter ineinanderlaufen. Schließlich prägt dieser Gebrauch von „Luhmann als Pop“ (Schäfer 2007) auch den Denkstil Goetz' eigener literarischer Texte. Im Abschlusstext seines Zyklus Festung, betitelt „Ästhetisches System“ (Goetz 1993, S. 365 ff.), wird die „Gesamtarchitektur“ des „Systems Goetz“ gar mit einem „Begehen der Luhmannschen Philosophie“ gleichgesetzt (vgl. Schäfer 2007, S. 263).

Dass dieser Sound auch mit Blick auf die affektive Praxis des Lesekollektivs erst im Vergleich zu anderen Theorien hervortritt, macht Goetz deutlich, wenn er Luhmann als „Antifasler unter den Träumern des Denkens“ bezeichnet (zit. n. Wegmann 2010, S. 469). Wohlwollend hebt er Luhmanns Klarheit und Purismus gleichermaßen vom Sound Kritischer Theorie wie der ,postmoderne[n] Theoretisiererei“ ab. Konkret heißt es: „Adorno im Vergleich zu ihm ein kompletter Wirrkopf, Foucault ein Märchenerzähler.“ (zit. n. ebd.) Auch Felsch sieht den „Charme der neuen Sachlichkeit“ (Felsch 2015, S. 233f.), der Luhmanns Leser:innen einnimmt, in dessen unwiderstehlicher „Coolness“ und „Eleganz“ begründet, die im Kontrast zum va-

\footnotetext{
13 Luhmann vergisst die Leser:in in seinen einleitenden Ausführungen zur Notwendigkeit einer abstrakten Darstellung in Soziale Systeme nicht, sondern fügt fast mitfühlend an, dass diese für die Leser:innenschaft eine in Kauf zu nehmende „Zumutung“ sei (Luhmann 1984, S. 13). Statt einer „Schnellstraße zum frohen Ende“ gleiche seine Theorieanlage eher einem „Labyrinth“ (ebd., S. 14). Entschließt sich die Leser:in also, den Weg durch das Abstraktionslabyrinth zu nehmen, ist das geduldige Ertragen dieser Zumutung gefragt. Was Luhmann mit seinem Fokus auf die formale und inhaltliche Struktur der Theorie jedoch entgeht, ist das affektive Vergnügen, das die begeisterte Theorieanhänger:in aus der Aufgabe schöpft, diese Abstraktheit zu bewältigen.
} 
gen Denkstil eines Deleuze gar ein „Pathos der Dezision“ auszustrahlen vermöge. Das exoterische Lesekollektiv aus Literat:innen und sonstigen interessierten Leser:innen nimmt so eine atmosphärische Kartierung der Theorie im Feld konkurrierender Denkstile vor.

Diese Abgrenzung findet auch in Deleuzes und Guattaris Abrechnung mit der Psychoanalyse und dem Marxismus in expliziter Form statt. Exoterische Lesekollektive knüpften daran an: Laut Manfred Frank wurde der Anti-Ödipus (Deleuze und Guattari 1977a),,im Geraune von Fan-Clubs und sektenähnlichen Gruppierungen am Rand der universitären Szene“ rezipiert (zit. n. Felsch 2015, S. 122). Zu diesem Lesekollektiv gehörte auch der Kreis der Merve-Verleger:innen, die in den 1970er-Jahren antraten, sich in einem Lektürekreis über fünf Jahre durch das erste gemeinsame Werk von Deleuze und Guattari zu arbeiten. Bei dem von Felsch erwähnten Anti-Ödipus-Lesekreis haben wir es mit einem exoterischen Suggestionskollektiv zu tun, das das Deleuze'sche Theorem der Intensität in eine intensive Leseatmosphäre übersetzte, das aber aus lauter Ehrfurcht vor dem Text offenbar die Aufforderung nonlinearen Lesens vergaß und stattdessen ernsthaft exegetisch vorzugehen versuchte. Relativ unabhängig von der Lektüre kultivieren Lesekreise dieser Art oftmals ein unbestimmtes Geheimnis, das nach innen die Botschaft hat: Immer wenn Du verstehst, verstehst Du falsch. ${ }^{14}$

Dass Kompliziertheit nicht das einzige Kriterium für die affektive Aufladung ist, zeigt die gleichzeitige Suche nach leichter Verständlichkeit. So entfaltete Deleuzes und Guattaris Rhizom (1977b) seine Beliebtheit in den ansonsten akademie- und theoriefernen Hippiezirkeln Westdeutschlands gerade weil es sich, wie Felsch beobachtet, ,,atmosphärisch lesen und intuitiv verstehen“ ließ (Felsch 2015, S. 115). Der alltagsnahe Atmosphärenbegriff, wie ihn Felsch hier verwendet, steht für einen ,leichten“, ja literarischen Lektüremodus. Wenn er von einer „Leseatmosphäre“ (ebd., S. 124) spricht, dann meint er die emphatisch-affektive Aufladung des kollektiven Lesens als potenziell bewusstseinserweiterndes Erlebnis. Der damit implizierte Atmosphärenbegriff ist jedoch vergleichsweise eng, da er vor allem auf Theorieemphase abzielt. Theorieatmosphären sollten dagegen im Sinne Flecks auch wissenschaftliche Denkstile erfassen, die der „Neutralität“ verpflichtet sind und möglicherweise eher langweilen als begeistern. Auch sie produzieren im Rahmen affektiver Praktiken Atmosphären, die sie mit den habituellen Regeln ihres Denkstils sowie ,gefühlsmäßiger Übereinstimmung“ (Fleck) aber erfolgreich unsichtbar machen. Für die Popularität von Theorien sind also nicht immer verhei-

\footnotetext{
14 Im McLuhan'schen Sinne lassen sich Theorielesekreise als Parainstitutionen verstehen, in denen Theorien „,vervollständigt“ werden. Mit mehr oder weniger Disziplin trifft sich das Denkkollektiv regelmäßig in Sitzungen von mehreren Stunden, um ein nicht selten schwieriges Werk kollektiv hermeneutisch zu erschließen. Nicht anders als in okkulten und religiösen Zirkeln lässt sich dabei das wiederholte laute Vorlesen schwieriger Sätze als affektive Praxis zur Herstellung einer Atmosphäre des Geheimnisvollen beobachten. Nicht von ungefähr werden Mainstream-Theorien von Theorieanhängern daher mit Skepsis, gar einem gewissen Ekel beäugt, geht ihnen doch das Verheißungsversprechen ab, das Davis (1971) zufolge für interessante Theorien unabdingbar ist. Letztere irritieren Leserannahmen demnach gekonnt und arbeiten mit gut dosierter Überraschung, während uninteressante Theorien vorhandene Annahmen bloß stützen. Interessant ist eine Theorie folglich, wenn sie sich nicht von selbst erklärt, sondern auf Enträtselung drängt, die Leser:in aber gerade mit so vielen Hinweisen und Erleuchtungsmomenten versorgt, dass sie am Ball bleibt und nicht kapituliert (ebd., S. 311).
} 
Bungsvolle deleuzianische Kryptik oder distanzerzeugender luhmannianischer Sachgehalt ausschlaggebend. Auch empirische Anwendbarkeit, politischer Gehalt oder (gegenwarts-)diagnostischer Wert können zum Ankerpunkt je spezifischer affektiver Praktiken werden.

\subsection{Dinge und Räume der Theorie}

Mit dem Lektürekreis ist ein organisatorischer Rahmen des exoterischen Denkkollektivs angesprochen, der auf die materielle Dimension des Lesens selbst, aber auch auf Dinge, Gegenstände und Infrastrukturen verweist. Dies lenkt den Blick auf die Materialität der ,Zwischenräume“, in denen Theorie zu zirkulieren beginnt. Felsch hat eine Reihe solcher Infrastrukturen im Westdeutschland der 1960er- bis 1980er-Jahre aufgezeigt: Verlage wie Merve und Suhrkamp wurden zu infrastrukturellen Knotenpunkten der kollektiven Theorierezeption, die in hohem Maße durch spezifische Umgebungen wie Kneipen und WG-Küchen beeinflusst wurde. Zur Infrastruktur gehörten auch Werkzeuge wie Notizbücher und Zettelkästen, die Leseund Schreibroutinen organisieren. Verlagstypische, auf hohen Wiedererkennungswert setzende Buchgestaltungen wie Suhrkamps stw-Reihe oder die Merve-Edition trugen wesentlich zu dieser Wirkung bei. In dieser wechselseitigen atmosphärischen Steigerung der stilspezifischen Wirkungen von Verlag und Autor profitierten beide Elemente voneinander.

Am atmosphärischen Erfolg von Luhmanns Denkstil hatte etwa der SuhrkampVerlag sicher einen wichtigen Anteil. Dieser bot Luhmanns Kritik der moralischpolitischen Überhitzung der Kritischen Theorie im Rahmen seiner - textlich und performativ ausgetragenen - Kontroverse mit Jürgen Habermas eine öffentlichkeitswirksame Arena (Habermas und Luhmann 1971). Wie Nikolaus Wegmann (2010, S. 565 ff.) argumentiert, ging Luhmanns durchschlagender Erfolg zu einem beträchtlichen Teil auf diese Kontroverse zurück. Nicht nur schärfte sich Luhmanns Profil, indem ihm der Status eines Antipoden des wesentlich bekannteren public intellectual Habermas zukam. Wichtiger noch: Luhmann, der vorher als Verwaltungswissenschafter und Soziologe in verschiedenen kleineren Verlagen in Erscheinung getreten war, erhielt plötzlich das „Format ,Theoretiker““ (ebd., S. 467) und wurde von Suhrkamp fortan als Hausautor behandelt.

Die affektive und energetische Aufladung der Streitform führte aber nicht zu einer Erhitzung des „,kühlen“ Luhmann. Zwar war sie förderlich, um Luhmanns Theorie zu einem Eigennamen - ja: zu einer Marke (Schimank 2012) - zu machen. Aber die Atmosphäre, die bis heute von diesem Namen ausgeht, bleibt mit den Attributen „kalt“, „technokratisch“ und „konservativ“ verbunden. Diese Akzentuierung ist zunächst kritisch, da aus der Position der Gegner:innen formuliert, öffnet sich aber gerade wegen ihres Provokationspotentials nicht nur für theoriepolitische, sondern auch für ästhetische Aufwertungen, etwa bei Goetz. So macht sich Luhmanns „Unterkühltheit“ nicht nur an der modularen Bauweise seiner Theorie und an ihrem Sound, sondern auch an seiner Selbstinszenierung im Rahmen von Interviews und Diskussionen fest, die häufig als reserviert, lakonisch und trocken-ironisch charakterisiert wird. Dieser auf Nicht-Performance angelegte Auftritt verdichtet sich zusammen mit dem auf Nicht-Sound angelegten Schreiben zur hochgradig faszinierenden 
Luhmann-Atmosphäre. Ihre Strahlkraft wird umso durchschlagender, je müheloser sie daherkommt. Der durch spezifische Formbildungen hergestellte Effekt der „Kälte" scheint nunmehr von der Autorität des Meisterdenkers selbst auszugehen. Hier beginnt die Mystifizierung einer „kalten“ Theorie. ${ }^{15}$

Verlage fungieren nicht nur als Infrastruktur bzw. als materieller „Behälter“ der Theorie, sondern lassen sich in ihrer Organisationsweise selbst von Theorieatmosphären affizieren. Das Merve-Verlagskollektiv um Peter Gente und Heidi Paris, das nicht zuletzt aufgrund der jahrelangen Lektüre des Anti-Ödipus eine Deleuzeaffinität entwickelt hatte, begann denn auch, die Vorgehensweise des Verlags am Denkstil Deleuzes auszurichten. ${ }^{16}$ Umgekehrt heftete sich die Theorieatmosphäre an die entsprechende „billige“ Buchgestaltung, die im Fall von Merves schlichtem buntem Raute-Design eine eigene Ikonizität, ja einen Kultwert erhielt. Dass der schmale Rhizom-Band von Deleuze und Guattari (1977b) in der olivgrünen Merve-Optik einen dekorativen Wert besitzt und sich - ohne notwendigerweise mit einer Lektüre verbunden zu sein - sowohl in Kunstbuchhandlungen als auch im Oberseminar gut macht, geht auf diese Verschmelzung von Autor und Verlag in einem ikonischen Ganzen zurück. Daran knüpfen sich affektive Praktiken des Sammelns von Gesamtund Erstausgaben, des Kuratierens der Büchersammlung und des Lesens an öffentlichen Plätzen wie Cafés und Parks - das Buchcover selbstverständlich gut sichtbar ausgestellt, um Insidern ein Erkennen zu ermöglichen.

Die Ästhetisierung und affektive Aufladung von Theorie funktioniert also nicht nur über das Lesen, sondern ist in ein Set affektiver Praktiken eingelassen, die performativ eingeübt werden. Dazu gehörte im ,langen Sommer der Theorie“, sich analog zum gutsortierten Plattenregal mit Bücherwänden aus bunten Merve- und Suhrkampbuchrücken zu umgeben, jeweils ein zerlesendes Exemplar in der Innentasche des Parkas mit sich herumzutragen und bestimmte Haltungen, Looks und Posen der großen Vorbilder zu imitieren, vom ständigen Rauchen über den Rollkragenpullover zum versonnenen Blick in die Ferne. Im Gegensatz zu den „Leseatmosphären“ im engeren Sinne ist das Lesen hier nur ein Element in einem Arrangement von Objekten und Artefakten. Theorie ist demnach nicht nur kognitive Aufgabe oder Lesevergnügen, sondern eine Lebenskunst. Auch Felschs Hitzemetapher in Der lange

\footnotetext{
15 So schlug Dirk Baecker in einem Anschreiben an den Merve-Verlag den Abdruck der gesammelten Interviews mit Luhmann vor und begründete dies damit, ,daß Luhmann einen sehr kühlen und ironischen, manchmal bissigen [...] Interviewstil entwickelt hat, der diesem Genre wieder etwas literarischen Schwung verleiht“ - mehr noch: diese Interviews könnten als „Einführungen in den spezifisch luhmannschen Theoriestil dienen“ (zit. n. Felsch 2015, S. 215). Diese Überzeugungsoffensive Baeckers, die die ästhetische Dimension der habituellen und performativen Selbstinszenierung Luhmanns (und ihren „Schwung“) mit seinem Theorie-, d.h. hier: seinem Denkstil kurzschließt, verweist auf die atmosphärenzentrierte Selektion theoretischer Texte beim Merve-Verlagskollektiv. Dass das Kollektiv sowohl die Interviews von Luhmann als auch die Arbeiten von Deleuze und Guattari herausgab, beweist sein ausgeprägtes Gespür für die Wirkweise von Theorieatmosphären. Die Denkstile beider Autoren(kollektive), Poststrukturalismus und Systemtheorie, sollten sich zu äußerst erfolgreichen Klassikern entwickeln, was zur Zeit des Erscheinens noch nicht abzusehen war.

16 Paris und Gente schrieben Deleuze in einem Brief, dass sie, angeregt von seiner Theorie des Minoritären, ,eine andere Art Verlag“ betrieben: „klein, billig, unscheinbar, daneben“ (zit. n. Felsch 2015, S. 109).
} 
Sommer der Theorie kündet davon, dass Theorie ab den 1960er-Jahren als exzessive, teils drogenähnliche Existenzweise praktiziert und inszeniert wurde. ${ }^{17}$

Dass Theorie zu einer Leidenschaft wird, die das Leben als Ganzes umfasst, hatte laut Diedrich Diederichsen mit ihrer lebenssteigernden Wirkung zu tun, in der das Sinnvolle und das Aufregende zusammenfielen (Diederichsen 2016, S. 125). Aufregend war und ist Theorie, weil sie Fragen der politischen Haltung, Fragen von Kunst und Pop mitverhandelte und mit ihrer Kraft, eine bestimmte Haltung zu erzeugen, so etwas wie Pop-Appeal entwickelte - ja, man kann sagen: Theorie funktioniert in ihrer Faszinationskraft, in ihrem Sound und ihren Verweissystemen bisweilen ähnlich wie Popkultur. Während ,Luhmann lesen“ von Goetz mit „Techno hören“ gleichgesetzt wird, empfiehlt Norbert Bolz, Deleuze zu lesen ,wie man ins Kino geht oder eine Platte hört“" (zit. n. Felsch 2015, S. 230). Nicht von ungefähr wurden Deleuze und Guattari in Kunstkontexten zu einer wichtigen Referenz.

Auch wenn die Faszinationskraft der Theorie, wie Felsch gezeigt hat, ihren historischen Höhepunkt in den 1980er-Jahren erreichte, büßte sie ihre lebensweltliche Attraktivität nach dem ,langen Sommer der Theorie“ nicht abrupt ein. Nachvollziehen lässt sich dies an den direkten Nachwirkungen der Goetz'schen Luhmann-Rezeption. Es ist eine Sache, dass sich eine Größe der Popliteratur mit abgebrochenem Soziologiestudium, dafür aber mit zwei Doktortiteln in Medizin und Geschichte, für Luhmanns Werk begeistert. Doch als Teil der Popkultur ist Goetz auch an dessen Zirkulation und Beschleunigung außerhalb der Wissenschaft beteiligt. Nicht zuletzt seine in die Romane eingestreuten Emphasen dürften dazu geführt haben, dass Luhmanns Gesellschaft der Gesellschaft 1998 auf der Spex-Bücherliste auf Platz 8 neben Pop-Literaten wie Nick Hornby und Max Goldt landete (Wegmann 2010, S. 469). Etwas Unwahrscheinliches trat ein: Luhmann wurde zum Pop-Accessoire.

\section{Schluss}

Dass Theorie zu Pop wird und sich ihre Strahlkraft nicht am Text, sondern an ihren „Simulakren“ festmacht, mag aus kulturkritischer Perspektive wie eine horrende Vorstellung anmuten. Der hier vorgestellte Ansatz hat jedoch zu zeigen versucht,

\footnotetext{
17 Folgerichtig beschrieb Foucault nach dem Erscheinen von Deleuzes und Guattaris Anti-Ödipus das Unterfangen, anti-ödipal zu sein, als ,Lebensstil [...], eine Art und Weise zu denken und so zu leben. Wie kann man sich davor bewahren, ein Faschist zu sein, auch wenn man sich für einen revolutionären Militanten hält? Wie können wir unser Sprechen und unser Tun, unsere Herzen und unsere Lüste vom Faschismus befreien? [...] Deleuze und Guattari verfolgen die leisesten Spuren des Faschismus im Körper.“ (zit. nach Bröckers 1992, S. 5) Es ist dabei kein Zufall, dass unter den Dingen der Theorie Drogen im „langen Sommer“ eine große Rolle spielten - sowohl mit Blick auf ihre affektiv-sensorischen Wirkungen auf die Theorieproduktion als auch auf die Lesepraktiken. Seien es die Unmengen Kaffee und Zigaretten, die während der Zusammenkünfte des Denkkollektivs „Lesekreis“ nicht nur die kognitive Aufmerksamkeit, sondern auch die sinnliche und affektive Empfänglichkeit für die Theorie schärften, seien es die Rauscherfahrungen durch andere Drogen, die, angeregt von der im Rhizom zu findenden Aussage: ,Wir haben halluzinatorische Erfahrungen gemacht“ (Deleuze und Guattari 1977b, S. 35), zur Erweiterung des theoretischen Horizonts in eine übersinnliche Sphäre eingenommen wurden. Luhmanns Empfehlung, die Konzentration beim Schreiben mithilfe von Schokolade und einem (!) abendlichen Schnaps zu erhöhen, nimmt sich dagegen wenig ekstatisch aus.
} 
dass die Atmosphäre ein konstitutiver Bestandteil von Theorien ist. Mehr noch: Es gibt, wie schon eingangs bemerkt, keine Theorie ohne Atmosphäre.

Zur Annäherung an das Phänomen der Theorieatmosphären ermöglicht die affekttheoretische Reformulierung der Fleck'schen Begriffe Denkstil und Denkkollektiv eine doppelgleisige Perspektive, die sich einerseits für das Wandern und andererseits für die Grenzbildungen in und durch Theorie interessiert. Die Unterscheidung von esoterischem und exoterischem Kreis hat sich als hilfreich erwiesen, um den ausschließenden Charakter von Denkkollektiven zu erfassen. Zugleich fungiert diese Unterscheidung als Impuls, um die in der Affekttheorie häufig ausgeblendeten Machteffekte von Atmosphären systematischer zu berücksichtigen. Atmosphären sind aus dieser Sicht keineswegs als ewiges dynamisches „Fließen“ aufzufassen, sondern folgen einer hochgradig exkludierenden Dynamik der Herstellung von Innen-Außen-Grenzen. Entgegen des häufigen Vorwurfs, wahlweise zu vage oder auch deterministisch zu sein, wurde die Theorieatmosphäre hier als affektive Praxis verstanden, die verbindende und trennende, öffnende und schließende, nomadische und begrenzende Effekte zeitigt.

Die Teilanalysen haben vorgeführt, wie die drei analytisch getrennten Dimensionen von Theorieatmosphären zusammenwirken. Der Theoriestreit wird nie nur von seinen Protagonisten im Text ausgetragen, sondern auf Bühnen und Podien, in Kritiken, Rezensionen und Kommentarspalten; er befördert die Zirkulation und das Branding von Theorien über den Text hinaus. Theorien erhalten qua so entstehender Markenzeichen eine affektiv aufgeladene Signatur - eine Atmosphäre. Das Beispiel der Kontroverse verweist dabei auf ein wesentliches Charakteristikum von Theorieatmosphären insgesamt: Sie wandern, und zwar in und durch Texte, über fremde disziplinäre Kontexte, hinein in neue, etwa literarische Textgattungen, in Räume und Milieus des Gebrauchs, der Praxis und der Aufführung. Im Nachvollzug dieser Wanderbewegungen wird die Stärke eines Atmosphärenbegriffs sichtbar, der die unvorhergesehenen Nachbarschaften, die durch räumliche Umgebungen hergestellt werden, innerhalb wie außerhalb des Textes lokalisiert. Denn die außertextlichen Formen und Räume - Kolloquien, Seminare, Lesekreise, Bibliotheken, Tagungen sind Bestandteile von Atmosphären, die entsprechend auch an der Entwicklung der Schulbildung beteiligt sind. Solche atmosphärischen Räume bringen geradezu Kunstformen hervor, aus denen heraus eine Theorierichtung als solche überhaupt erst Kontur gewinnt.

Erst aus dem Zusammenspiel der drei Dimensionen von Theorieatmosphären werden exemplarisch die konträren Denkstile der Systemtheoretiker:innen und der Deleuzianer:innen deutlich: Ein überlegenes Distanzpathos der Beobachtung zweiter Ordnung steht gutgelaunter Affirmation gegenüber. Hier handelt es sich weder nur um einen habituellen Unterschied noch um Sekundärfolgen eines Theoriesounds. Neben der Lesepraxis müssen auch die Rolle des materiellen Textmediums und der Dinge und Räume in ihrem wechselseitigen Verhältnis in Rechnung gestellt werden. Atmosphärische Rezeption kann dann nicht länger mit der emphatischen Lektüre auratischer Texte gleichgesetzt werden, sondern umfasst neben dem pragmatischen oder gelangweilten Lesen auch diverse außertextliche Aspekte - wie etwa im Fall Merve gesehen. Zwar sind die beleuchteten Beispiele in ihrem historischen Kontext und in der Auswahl der akademischen Milieus spezifisch und 
können nicht ohne weiteres generalisiert werden. Der angesprochene Komplex der westdeutschen Theorierezeption nach 1968 hat jedoch den Vorteil, dass in ihm die Theorieatmosphären in all ihren zentralen Facetten sehr deutlich hervortreten. Ein komplizierteres, aber zweifellos lohnendes Unterfangen wäre sicher die Erweiterung der Atmosphärenanalyse auf weniger auratisierte und soziologiehistorisch weniger beachtete Theorien.

Der Atmosphärenbegriff lädt dazu ein, über die Verschiebungen und Rekonfigurationen der theoretischen Milieus nachzudenken, die sich seit dem Ende der Großtheorien ergeben haben. Man mag bei einem Blick auf die heutige Theoriebildung in der Soziologie geneigt sein, eine zunehmend lauwarme Tendenz zur leichten Verständlichkeit, zu griffigen Thesen und einem unaufgeregt-professionellen Sound zu verzeichnen. Die große Flut an Einführungs- und Lehrbuchtheorie nimmt der Leser:in die Vervollständigungsarbeit durch didaktische Anmoderation, die Verwendung eingängiger Beispiele, lehrbuchartige Zwischenresümees und schematische Aufzählungen zu einem beträchtlichen Teil ab. Die großen Theoriestreits sind zunehmend Debatten über die richtige Methode gewichen. Nicht zuletzt steht heutige Theorieproduktion unter einem stärkeren Druck hinsichtlich Drittmitteleinwerbung, Journal-Publikationen und der generellen Rechtfertigung ihrer außerakademischen Relevanz. ${ }^{18}$

Doch nur in der Begrifflichkeit des Rück- und Untergangs von den neuen Theorieatmosphären zu sprechen, wäre fatal - nicht nur, weil der Rückgang der Autorität (männlicher) Meisterdenker überfällig war, sondern weil die neuartigen Formen der Theorieatmosphären so im Dunkeln blieben. So hat sich gerade im englischsprachigen Raum der Theorieblog als wichtiges Format eines eher spielerischen und essayistischen Schreibens von und über Theorie entwickelt. Die Blogs von Graham Harman oder Timothy Morton etwa haben den Denkstil der „Object-Oriented Ontology“ (OOO) in seiner Entstehung maßgeblich geprägt. Auf Facebook erfreut sich die Gruppe „Luhmanns Humor“ großer Beliebtheit; und auch die Theoriemilieus auf Twitter, die kleine Fundstücke, Textpassagen, CfPs und Anekdoten aus dem Institutsalltag teilen, sind nicht von einer ernsthaft-emphatischen, sondern von einem teils hochgradig ironischen, ja albernen Umgang mit diesen Referenzen geprägt. Wer sich auf die Suche nach Memes oder Motto-T-Shirts mit Bezug auf Foucault, Adorno, Marx, Deleuze, Luhmann, Butler und Žižek macht, wird von der Fülle regelrecht erschlagen. Bemerkenswerterweise sind es hier doch tendenziell wieder die Klassiker:innen, die für solche popkulturellen Aufladungen geeignet sind.

\footnotetext{
18 Anzeichen für eine gewisse „Abkühlung“ im Vergleich zum ekstatischen „Theoriesommer“ sind zumindest mit Blick auf die Soziologie der Bedeutungsverlust der Großtheorien und ihrer lebenssteigernden Wirkung sowie eine deutliche Abschwächung des auf Charisma basierenden Meisterdiskurses. Seltsam aus der Zeit gefallen wirken heute reine Vertreter:innen der einzelnen Theorieschulen. Juvans (2018) These dazu lautet, dass dieses Charisma der Theorie, das seinen historischen Höhepunkt in der Mitte des 20. Jahrhunderts erreichte, in der Gegenwart durch ein globales Starsystem der Theorie ersetzt wird, das sich an den transnationalen Publikationsstrategien US-amerikanischer Universitäten orientiert. Während Juvan ein Ende des Theoriecharismas konstatiert, ist aus der hier vertretenen Perspektive gleichwohl kein Ende in Sicht: Solange Theorie produziert und rezipiert wird, ist eine Theorieatmosphäre, so unscheinbar sie sein mag, immer schon vorhanden.
} 
Mit Blick auf die lebenden Popstars der Theorie ist das Format des Events auf dem Vormarsch, sei es auf großen Theaterbühnen, Festivals und Science Slams, bei denen es mitunter zu einer Reaktivierung der ehrfurchtsvollen Salonatmosphäre kommt, die aber auch auf Nahbarkeit und Unterhaltung setzen. Theorie wird so zu einem immer selbstverständlicheren Teil der Popkultur. Zur Befürchtung, dass die Buchform in einem solchen Gebrauch von Theorie untergeht, besteht allerdings kein Anlass: Nachdem das Model Gigi Hadid mit einem Camus-Buch und der Sänger Harry Styles mit einem Exemplar von Susan Sontags Against Interpretation gesichtet wurden, versicherte die New York Post, das Buch sei das „hot new accessory of 2019“ (Laneri 2019).

Funding Open Access funding enabled and organized by Projekt DEAL.

Open Access Dieser Artikel wird unter der Creative Commons Namensnennung 4.0 International Lizenz veröffentlicht, welche die Nutzung, Vervielfältigung, Bearbeitung, Verbreitung und Wiedergabe in jeglichem Medium und Format erlaubt, sofern Sie den/die ursprünglichen Autor(en) und die Quelle ordnungsgemäß nennen, einen Link zur Creative Commons Lizenz beifügen und angeben, ob Änderungen vorgenommen wurden.

Die in diesem Artikel enthaltenen Bilder und sonstiges Drittmaterial unterliegen ebenfalls der genannten Creative Commons Lizenz, sofern sich aus der Abbildungslegende nichts anderes ergibt. Sofern das betreffende Material nicht unter der genannten Creative Commons Lizenz steht und die betreffende Handlung nicht nach gesetzlichen Vorschriften erlaubt ist, ist für die oben aufgeführten Weiterverwendungen des Materials die Einwilligung des jeweiligen Rechteinhabers einzuholen.

Weitere Details zur Lizenz entnehmen Sie bitte der Lizenzinformation auf http://creativecommons.org/ licenses/by/4.0/deed.de.

\section{Literatur}

Abbott, A. (2004). Methods of discovery: Heuristics for the social sciences. New York: W. W. Norton \& Co.

Anderson, B. (2009). Affective atmospheres. Emotion, Space and Society, 2(2), 77-81.

Anz, T. (2010). Niklas Luhmanns rätselhaftes Gastspiel im Zentrum Kritischer Theorie. Über eine abgebrochene Spurensuche - mit einer Nachbemerkung zu Jürgen Habermas' Stil wissenschaftlicher Kommunikation. URL: https://literaturkritik.de/public/rezension.php?rez_id=13166. Zugegriffen: April 2021.

Bartmanski, D. (2012). How to become an iconic social thinker. The intellectual pursuits of Malinowski and Foucault. European Journal of Social Theory, 15, 426-452.

Bauer, J. (2014). „Gerichtetes Wahrnehmen“, „Stimmung“, „,soziale Verstärkung“. Zur historischen Semantik einiger Grundbegriffe der „Lehre vom Denkstil und Denkollektiv“. NTM. Zeitschrift für Geschichte der Wissenschaften, Technik und Medizin, 22, 87-109.

Bille, M., Bjerregaard, P., \& Sørensen, T. F. (2015). Staging atmospheres: Materiality, culture, and the texture of the in-between. Emotion, Space and Society, 15, 31-38.

Bissell, D. (2010). Passenger mobilities: Affective atmospheres and the sociality of public transport. Environment and Planning, 28, 270-289.

Böhme, G. (1995). Atmosphäre. Essays zur neuen Ästhetik. Frankfurt a. M.: Suhrkamp.

Böhme, G. (2007). Atmosphären wahrnehmen, Atmosphären gestalten, mit Atmosphären leben: Ein neues Konzept ästhetischer Bildung. In R. Goetz \& S. Graupner (Hrsg.), Atmosphäre(n). Interdisziplinäre Annährungen an einen unscharfen Begriff (S. 31-44). München: Kopaed.

Bröckers, M. (1992). Und... und... und. „Tausend Plateaus“ von Gilles Deleuze und Felix Guattari. taz. am Wochenende vom 31. Okt. 1992. URL: https://taz.de/!1645739/. Zugegriffen: April 2021.

Brown, S. D., Kanyeredzi, A., McGrath, L., Reavey, P., \& Tucker, I. (2019). Affect theory and the concept of atmosphere. Distinktion. Journal of Social Theory, 20, 5-24. 
Culp, A. (2015). Dark Deleuze. URL: https://non.copyriot.com/dark-deleuze/. Zugegriffen: April 2021.

Davis, M. S. (1971). That's interesting! Towards a phenomenology of sociology and a sociology of phenomenology. Philosophy of the Social Sciences, 1, 309-344.

Deleuze, G., \& Guattari, F. (1977a). Anti-Ödipus. Kapitalismus und Schizophrenie I. Frankfurt a. M.: Suhrkamp.

Deleuze, G., \& Guattari, F. (1977b). Rhizom. Berlin: Merve.

Deleuze, G., \& Guattari, F. (1992). Tausend Plateaus. Kapitalismus und Schizophrenie II. Berlin: Merve.

Delitz, H. (2015). Bergson-Effekte. Aversionen und Attraktionen im französischen soziologischen Denken. Weilerswist: Velbrück.

Diederichsen, D. (2016). Es ist spät geworden. Zeitschrift für Kulturwissenschaften, 10, 125-127.

Edensor, T. (2015). Producing atmospheres at the match: Fan cultures, commercialisation and mood management in English football. Emotion, Space and Society, 15, 82-89.

Egloff, R. (2005). Einleitung. In R. Egloff (Hrsg.), Tatsache-Denkstil-Kontroverse. Auseinandersetzungen mit Ludwik Fleck (S. 7-12). Zürich: Collegium Helveticum.

Engert, K., \& Krey, B. (2013). Das lesende Schreiben und das schreibende Lesen. Zur epistemischen Arbeit an wissenschaftlichen Texten. Zeitschrift für Soziologie, 42, 366-384.

Farzin, S. (2011). Die Rhetorik der Exklusion. Zum Zusammenhang von Exklusionsthematik und Sozialtheorie. Weilerswist: Velbrück.

Farzin, S., \& Laux, H. (Hrsg.). (2014). Gründungsszenen soziologischer Theorie. Wiesbaden: Springer VS.

Felsch, P. (2015). Der lange Sommer der Theorie. Geschichte einer Revolte 1960-1990. Frankfurt a. M.: Fischer.

Fleck, L. (1980 [1935]). Entstehung und Entwicklung einer wissenschaftlichen Tatsache. Einführung in die Lehre vom Denkstil und Denkkollektiv. Frankfurt a. M.: Suhrkamp.

Galloway, A. (2015). Forget Deleuze! URL: http://cultureandcommunication.org/galloway/forget-deleuze. Zugegriffen: April 2021.

Galloway, A. (2018). Theory Hot and Cold. URL: http://cultureandcommunication.org/galloway/theoryhot-and-cold. Zugegriffen: April 2021.

Genette, G. (1989). Paratexte: Das Buch vom Beiwerk des Buches. Frankfurt a. M.: Campus.

Goetz, R. (1993). Ästhetisches System. In R. Goetz, Kronos. Berichte (S. 365-401). Frankfurt a. M.: Suhrkamp.

Goetz, R. (1999). Abfall für alle. Roman eines Jahres. Frankfurt a. M.: Suhrkamp.

Goetz, R. (2001). Rave. Erzählung. Frankfurt a. M.: Suhrkamp.

Groebner, V. (2012). Wissenschaftssprache. Eine Gebrauchsanweisung. Konstanz: Konstanz University Press.

Gugutzer, R. (2020). Beyond Husserl and Schütz. Hermann Schmitz and neophenomenological sociology. Journal for the Theory of Social Behaviour, 50, 184-202.

Gumbrecht, H. U. (2011). Stimmungen lesen. Über eine verdeckte Wirklichkeit der Literatur. München: Hanser.

Habermas, J., \& Luhmann, N. (1971). Theorie der Gesellschaft oder Sozialtechnologie. Was leistet die Systemforschung? Frankfurt a. M.: Suhrkamp.

Juvan, M. (2018). The charisma of theory. Textxet: Studies in Comparative Literature, 86, 89-110.

Kalthoff, H. (2008). Einleitung: Zur Dialektik von qualitativer Forschung und soziologischer Theoriebildung. In H. Kalthoff, S. Hirschauer \& G. Lindemann (Hrsg.), Theoretische Empirie. Zur Relevanz qualitativer Forschung (S. 8-34). Frankfurt a. M.: Suhrkamp.

Kuhn, T. S. (1962). The structure of scientific revolutions. Chicago: University of Chicago Press.

Laneri, R. (2019). Bella and Gigi Hadid make books the hot new accessory of 2019. New York Post vom 19. März 2019. URL: https://nypost.com/2019/03/19/bella-and-gigi-hadid-make-booksthe-hot-new-accessory-of-2019/?utm_campaign=SocialFlow\&utm_medium=SocialFlow\&utm_ source=NYPTwitter. Zugegriffen: April 2021.

Lierschof (2017). Tagungsprotokoll zum 90sten von Niklas Luhmann am 08/09.12.2017 - eine systemische Satire! URL: https://luhmannsschwarzehefte.wordpress.com/2017/12/16/tagungsprotokollzum-90ste-von-niklas-luhmann-am-08-09-12-2017-eine-systemische-satire/. Zugegriffen: April 2021.

Löw, M. (2001). Raumsoziologie. Frankfurt a. M.: Suhrkamp.

Lüdemann, S. (2004). Metaphern der Gesellschaft. Studien zum soziologischen und politischen Imaginären. München: Wilhelm Fink.

Luhmann, N. (1982). Liebe als Passion. Zur Codierung von Intimität. Frankfurt a. M.: Suhrkamp.

Luhmann, N. (1984). Soziale Systeme. Grundriß einer allgemeinen Theorie. Frankfurt a. M.: Suhrkamp.

Luhmann, N. (2008). Liebe. Eine Übung. Frankfurt a. M.: Suhrkamp. 
Massumi, B. (2011). Semblance and event. Activist philosophy and the occurrent arts. Cambridge (USA): MIT Press.

McCormack, D. P. (2008). Engineering affective atmospheres on the moving geographies of the 1897 Andrée expedition. Cultural Geographies, 15, 413-430.

McLuhan, M. (1964). Understanding media. London: Routledge.

Rasch, W., \& Knodt, E. M. (1994). Systems theory and the system of theory. New German Critique, 61, 3-8.

Rauh, A. (2012). Die besondere Atmosphäre. Ästhetische Feldforschungen. Bielefeld: transcript.

Reckwitz, A. (2016). Praktiken und ihre Affekte. In H. Schäfer (Hrsg.), Praxistheorien. Ein soziologisches Forschungsprogramm (S. 163-180). Bielefeld: transcript.

Rheinberger, H.-J. (2005). Ludwik Fleck und die Historizität wissenschaftlichen Wissens. In R. Egloff (Hrsg.), Tatsache - Denkstil - Kontroverse. Auseinandersetzungen mit Ludwik Fleck (S. 29-32). Zürich: Collegium Helveticum.

Riedel, F. (2015). Music as atmosphere: Lines of becoming in congregational worship. Lebenswelt, 6, $80-111$.

Riedel, F. (2019). Atmosphere. In J. Slaby \& C. von Scheve (Hrsg.), Affective societies. Key concepts (S. 85-95). Abingdon-on-Thames: Routledge.

Salter, C. (2014). Atmospheres of affect. In M.-L. Angerer (Hrsg.), Timing of affect. Epistemologies, aesthetics, politics (S. 225-243). Zürich: Diaphanes.

Schäfer, M. J. (2007). Luhmann als „Pop“. Zum „ästhetischen System“ Rainald Goetz’. In C. Huck \& C. Zorn (Hrsg.), Das Populäre der Gesellschaft. Systemtheorie und Populärkultur (S. 262-283). Wiesbaden: VS Verlag für Sozialwissenschaften.

Schäfers, L., \& Schnelle, T. (1980). Ludwik Flecks Begründung der soziologischen Betrachtungsweise in der Wissenschaftstheorie. Einleitung. In L. Fleck, Entstehung und Entwicklung einer wissenschaftlichen Tatsache. Einführung in die Lehre vom Denkstil und Denkkollektiv (S. VII-XLIX). Frankfurt a. M.: Suhrkamp.

Schimank U. (2012). Markenbildung und Markenbindung auf dem Theorie-Markt. Eine Notiz zur Soziologie der Soziologie. Zeitschrift für Theoretische Soziologie, 1, 10-15.

Schlechtriemen, T. (2014). Bilder des Sozialen. Das Netzwerk in der soziologischen Theorie. Paderborn: Wilhelm Fink.

Schmitz, H. (2007). Der Leib, der Raum und die Gefühle. Bielefeld: Edition Sirius.

Schmitz, H. (2012). Atmosphäre und Gefühl. Für eine neue Phänomenologie. In C. Heibach (Hrsg.), Atmosphären. Dimensionen eines diffusen Phänomens (S. 39-56). München: Wilhelm Fink.

Seyfert, R. (2012). Atmosphären - Transmissionen - Interaktionen: Zu einer Theorie sozialer Affekte. Soziale Systeme, 17, 73-96.

Seyfert, R. (2019). Beziehungsweisen. Elemente einer relationalen Soziologie. Weilerswist: Velbrück.

Slaby, J. (2018). Drei Haltungen der Affect Studies. In L. Pfaller \& B. Wiesse (Hrsg.), Stimmungen und Atmosphären. Zur Affektivität des Sozialen (S. 53-82). Wiesbaden: Springer VS.

Slaby, J. (2019). Affective arrangement. In J. Slaby \& C. von Scheve (Hrsg.), Affective societies. Key concepts (S. 109-118). Abingdon-on-Thames: Routledge.

Swedberg, R. (2014). The art of social theory. Princeton: Princeton University Press.

Thibaud, J.-P. (2011). The sensory fabric of urban ambiances. The Senses and Society, 6, 203-215.

Thrift, N. (2008). Non-representational theory. Space, politics, affect. London, New York: Routledge.

Wagner, G. (2007). Die Atmosphäre der Kunstwelt: Arthur C. Danto, Andy Warhol und die Soziologie der PopArt. In R. Goetz \& S. Graupner (Hrsg.), Atmosphäre(n). Interdisziplinäre Annährungen an einen unscharfen Begriff (S. 143-162). München: Kopaed.

Weber, M. (1980). Wirtschaft und Gesellschaft. Grundriß der verstehenden Soziologie. 5., rev. Aufl. Tübingen: Mohr.

Wegmann, N. (2010). Wie kommt die Theorie zum Leser? Der Suhrkamp Verlag und der Ruhm der Systemtheorie. Soziale Systeme, 16, 463-470.

Werber, N. (2000). Benjamin, remixt. Dichter mittleren Alters lasen Suhrkamp-Denker in Hamburg. URL: http://homepage.ruhr-uni-bochum.de/niels.werber/lesung.htm. Zugegriffen: April 2021.

Wetherell, M. (2013). Feeling rules, atmospheres and affective practice: Some reflections on the analysis of emotional episodes. In C. Maxwell \& P. Aggleton (Hrsg.). Privilege, agency and affect (S. 221-239). New York: Palgrave Macmillan. 
Elena Beregow geb. 1989. Wissenschaftliche Mitarbeiterin am Lehrstuhl für Allgemeine Soziologie der Universität der Bundeswehr München. Forschungsschwerpunkte: Soziologische Theorie, Medien- und Kultursoziologie, Popkultur, Metaphern, Neue Materialismen. Ausgewählte Veröffentlichungen: Fermente des Sozialen. Thermische Figuren in der Sozialtheorie, 2021; Cooked or Fermented? The Thermal Logic of Social Transformation, in: Culture Machine, 2018; Nichts dahinter - Pop-Oberflächen nach der Postmoderne, in: Pop. Kultur und Kritik, 2018. 